\title{
Experiment and Simulation of the Fabrication Process of Lithium-ion Battery Cathodes for Determining Microstructure and Mechanical Properties
}

\author{
Mehdi Forouzan ${ }^{\mathrm{a}}$, Chien-Wei Chao ${ }^{\mathrm{a}}$, Danilo Bustamante ${ }^{\mathrm{b}}$, Brian A. Mazzeo ${ }^{\mathrm{b}}$, Dean R. Wheeler ${ }^{\mathrm{a} *}$ \\ ${ }^{a}$ Department of Chemical Engineering, Brigham Young University, Provo, UT 84602, USA \\ ${ }^{\mathrm{b}}$ Department of Electrical \& Computer Engineering, Brigham Young University, Provo, UT 84602, \\ USA \\ * Corresponding author. Tel.: +1 801422 4126; fax: +1 8014220151. \\ E-mail address: dean_wheeler@byu.edu (D.R. Wheeler).
}

\begin{abstract}
The fabrication process of Li-ion battery electrodes plays a prominent role in the microstructure and corresponding cell performance. Here, a mesoscale particle dynamics simulation is developed to relate the manufacturing process of a cathode containing Toda NCM-523 active material to physical and structural properties of the dried film. Particle interactions are simulated with shifted-force LennardJones and granular Hertzian functions. LAMMPS, a freely available particle simulator, is used to generate particle trajectories and resulting predicted properties. To make simulations of the full film thickness feasible, the carbon binder domain (CBD) is approximated with $\mu$ m-scale particles, each representing about 1000 carbon black particles and associated binder. Metrics for model parameterization and validation are measured experimentally and include the following: slurry viscosity, elasticity of the dried film, shrinkage ratio during drying, volume fraction of phases, slurry and dried film densities, and microstructure cross sections. Simulation results are in substantial agreement with experiment, showing that the simulations reasonably reproduce the relevant physics of particle arrangement during fabrication.
\end{abstract}

Keywords: Li-ion cathode; particle dynamics simulation; microstructure prediction; slurry viscosity; 
electrode elasticity.

\section{Introduction}

\subsection{The importance of microstructure}

For batteries that contain porous electrodes, the electrode microstructure plays a pivotal role in determining cell performance $[\underline{1}, \underline{2}]$. This is due to the fact that effective transport parameters, namely ionic conductivity, electronic conductivity, and diffusivity, are determined by the structure of species pathways through the porous electrode. Ions are generally transported through the electrolyte-filled pores, so the porosity and tortuosity are key variables that determine the ionic conductivity and diffusivity [3]. Likewise, active materials frequently are electronically semiconductive, so conductive additive (usually carbon) is often included to improve electronic conductivity [4-6]. Unfortunately, such additives can increase tortuosity and decrease porosity of the electrodes, resulting in reduction of ionic conductivity [7-10]. Likewise, the degree to which active materials are coated by conductive carbon is known to affect cell performance $[9,11]$. All of these factors illustrate how electrochemical performance is influenced by microstructure, which in turn is dependent on fabrication parameters and cell history.

Commercial lithium-ion battery electrodes are fabricated through four main steps: mixing, coating, drying, and calendering. During the mixing step, the dry components of an electrode (carbon black, binder, and active material) are generally mixed with solvent (commonly NMP) to form a slurry. In the coating step the slurry is applied to a metal foil current collector and smoothed to a uniform film thickness. The resulting film is then heated to evaporate the solvent. Subsequently the film is calendered (compressed between metal rollers) to a desired thickness to achieve desired porosity and to enhance electronic contacts. Following these steps, the cathode composite film, anode composite film, 
separator, and electrolyte are hermetically packaged to make an electrochemical cell [12-14].

Specific instances of the above general fabrication process are not necessarily optimal, and prudent modifications could increase cell performance even with existing materials. Many fabrication factors affect electrode microstructure including the composition (type and amount of active and carbon particles and binder), mixing sequence, ratio of solvent, temperature of the slurry, coating rate, coating thickness, drying procedure, and degree of calendering $[\underline{9}, \underline{11}, \underline{12}, \underline{14-24]}$. An optimization process on these and other variables is aided by knowledge of the relationships between the fabrication process, microstructure, and performance of the battery, which is quite challenging to obtain. Understanding the electrode microstructure is key to making such connections between fabrication steps and battery performance. Microstructures can be produced both by experiment and by modeling.

\subsection{Microstructure measurement}

X-ray computed tomography (XCT) and focused ion beam (FIB)/ scanning electron microscope (SEM) imaging are increasingly common techniques to determine 3D microstructure of porous electrodes [25-33]. Generally, a wide range of resolutions for XCT are possible, depending on the energy of the X-rays. For instance, so-called $\mu \mathrm{CT}$ with resolution around 1-2 $\mu \mathrm{m}$ and transmission $\mathrm{X}$ ray microscopy (TXM) with resolution around $50 \mathrm{~nm}$ are possible. Although XCT is a nondestructive method, it is generally challenging to differentiate the carbon and binder from the pores by $\mu \mathrm{CT}$ due to carbon's relatively small amount of X-ray absorption $[\underline{9}, \underline{10}, \underline{34}]$. Therefore, $\mu \mathrm{CT}$ is better-suited to identifying locations and shapes of the active material only. However, a few works have used TXM or synchrotron X-ray nano-tomography to distinguish between carbon, binder, and pores with high resolution (sub-50 nm) [32, 33]. As for FIB/SEM, it permits identification of the carbon-binder domain, but imaging a large-enough reconstruction volume to be representative (e.g. $50 \mu \mathrm{m} \times 50 \mu \mathrm{m}$ $\times 50 \mu \mathrm{m})$ is time-consuming, not to mention FIB/SEM tomography is by its nature a destructive method 
[29, 30, 35-38]. While much progress has been made in using both of these imaging techniques, they remain expensive for routine use. Nor can they be used for iterative optimization of the microstructure. Therefore we seek a computational model that can accurately predict the microstructure of typical particle-based battery electrodes.

\subsection{Microstructure prediction}

A realistic microstructure-prediction model could reduce reliance on experiments, allow exploration of as-yet-unrealized materials and processes, and yield physical insight during the optimization process. Also, realistic microstructures are needed as inputs to other battery models that simulate cell cycling and degradation.

Statistical approaches have been previously used to predict and reconstruct the microstructure of porous electrodes $[10, \underline{39}, \underline{40}]$. Such methods are based on reproducing experimental structures or parameters in a statistical sense, without regard for the process by which those structures were made in the real world. Stephenson et al. devised the stochastic grid (SG) model to understand the microstructure and transport properties of Li-ion electrodes based on a limited amount of experimental input [7]. One of the key advances of this approach, which was based on experimental observations and computational need, was to combine nanoscale carbon, binder, and pores into what is termed the carbon-binder domain (CBD). The $\mathrm{CBD}$ is distinct from the active material and $\mu \mathrm{m}$-scale pores, such that complete porous electrodes can be modeled as a three-phase system.

In a hybrid-statistical approach, Zielke et al. employed synchrotron-based nCT to image the active phase. Then they tried two statistical models, a random-cluster model and a fiber model, in one work [9] and the SG model in another work [10] to predict the locations and structure of the CBD. In more recent work, SEM/FIB was used to analyze the nanostructure of the CBD [요] . These novel combinations of experiment and model demonstrate the considerable effect of carbon-binder domain 
morphology and composition on transport parameters.

The main objective of this work is to develop a particle-based simulation to better understand the connection between the fabrication process and microstructure. The model is intended to reproduce the physics of particle-to-particle interactions while using a targeted amount of coarse-graining to make the model computationally feasible. LAMMPS, a freely available particle simulator, was used. In addition to predicting microstructure for a given condition, mechanical and structural properties like viscosity of slurry, elasticity of the dried film, and volume fraction of each phase are computed. Though we eventually intend to use this model to examine all the steps of the fabrication process, this paper focuses on method development and on the characteristics of the film before and after the drying process. The film experiences a large volume change and dislocation of solid materials during drying, making this change a key factor in the resulting microstructure and electrochemical parameters [23].

The model development was divided into two parts for more robust parameterization. First, a purecarbon (no active material) model was developed, parameterized, and validated. Then, active particles were added and simulations and experiments were repeated in order to determine parameters specific to active material. For both the carbon model and the active model, regression of the parameters was performed semi-empirically by comparing to experimental results that included: slurry viscosity at shear rates ranging from $1 \mathrm{~s}^{-1}$ to $1100 \mathrm{~s}^{-1}$, shrinkage ratio during drying, film elasticity, film density, and volume fractions of constituent phases. Finally, a 2D image of cross-section of the real electrode was compared with one from the simulation in each case.

\section{Fabrication Process Simulation}

As discussed above, the fabrication process is key to understanding the microstructure of pristine electrodes and initial performance of the battery. However, not much is understood about how the 
particles self-organize during the slurry-making, drying and calendering processes and how these affect cell performance. Although experimental procedures could be developed to address these issues, they are currently lacking. In any case, techniques such as synchrotron-based nCT are expensive and fundamental modeling methods can partially meet this need.

Multiple researchers have studied electrode fabrication and microstructure in fundamental ways. Li et al. reviewed material processing techniques for fabrication of constituent of Li-ion batteries [19]. Liu et al. studied the effect of different methods of mixing [16]. Haselrieder et al. assessed the impact of the calendering process on the structure and performance of Li-ion batteries in terms of surface morphology and mechanical, structural and electrochemical properties [22]. Though not specifically for battery electrodes, a drying model of nanoparticles in solvent was developed by Cheng and coworkers [41].To the best of our knowledge, previous work does not include a model that includes multiple fabrication processes, including the drying process, on the length scale of 1-100 $\mu \mathrm{m}$.

The following subsections describe the main ideas of the particle-based fabrication model. Simulation details are given as well.

\subsection{Modeling Approaches}

Recently Liu et al. developed a 2D coarse-grained kinetic Monte Carlo (KMC) simulation to investigate the effect of mixing sequences, drying conditions, and active particle morphology on microstructure and performance $[\underline{11}, \underline{18}]$.

Similarly, Cerbelaud et al. developed a Brownian dynamics model for simulating the microstructure of a silicon anode from first principles [42]. Brownian dynamics, as a particle simulation technique, uses an implicit solvent and nanoscale particles. Potential functions between pairs of particles incorporate the mediating effect of the solvent. Cerbelaud et al. showed how individual carbon black 
particles $(50 \mathrm{~nm}$ diameter) and nanoscale silicon particles $(50-150 \mathrm{~nm}$ diameter) form aggregates. However, due to the number of particles feasible to simulate, achieving this resolution means the length of the simulation box accessible to this technique is approximately $1 \mu \mathrm{m}$.

Here we present a new approach to predict the microstructure of slurry and dried film for different fabrication conditions through a particle-based dynamic simulation. Like Brownian dynamics, solvent is treated implicitly and particles interact through pairwise forces. However, our objective is to simulate a much larger length scale, namely the full thickness of the electrode film $(30-80 \mu \mathrm{m})$. Like all particle dynamics techniques, the simulation can make it possible to observe evolution of the structure over time, in this case during the coating and drying processes.

A preliminary version of this model was published by our group previously [7], which we term DPP1 (DPP = dynamic particle packing). The previous model used Lennard-Jones forces to imitate elastic (conservative) interparticle collisions. The model was capable of generating the microstructure of the finished electrode, but did not attempt to deal with specific fabrication processes. Structural metrics were utilized for parametrization of the model. A key aspect of the DPP1 model (and the present work) is to treat the carbon-binder domain by a superposition of so-called CBD particles. One $\mu \mathrm{m}$-scale CBD particle represents a particular mass of carbon and binder in a particular volume.

The present model, which we term DPP2, considers additional forces on mesoscale particles including contact friction and damping forces due to solvent. This more realistic treatment allows us to predict reasonable microstructures of the slurry and dried film. The required additional force parameters are regressed in a semi-empirical fashion by comparing simulation and experimental properties, many of which do not have standard values in the literature. Thus, a significant part of this work is the determination of fundamental experimental properties that have bearing on electrode fabrication and microstructure. 


\subsection{Potential Functions}

In order to determine particle motion and configurations, pairwise potential or force functions are necessary. Here we use the shifted-force Lennard-Jones (LJ/SF) potential function and the granular Hertzian $(\mathrm{GH})$ force, both of which are built into the LAMMPS particle simulation program.

The LJ/SF potential contains both attraction and repulsion and has zero force and potential at a particular cut-off length $\left(r_{\mathrm{c}}\right)$ beyond which no interaction is computed. This potential function is particularly needed to represent the interaction between particles in the solid when more attraction is necessary. Because particle dynamics simulations are based on solving Newton's equations of motion and the time-consuming stage is the calculation of the forces, LJ/SF potential allows lower computational cost compared to the traditional Lennard-Jones (LJ) potential, due to the ability to use smaller values of $r_{\mathrm{c}}$ without harming numerical stability.

Two parameters are required in the LJ potential function: $\sigma$ and $\varepsilon$, which represent the size of the particle and the attractive energy well depth, respectively. The form of this potential, $U_{\mathrm{LJ}}$, is [7]

$$
U_{\mathrm{LJ}}(r)=4 \varepsilon\left[\left(\frac{\sigma}{r}\right)^{12}-\left(\frac{\sigma}{r}\right)^{6}\right]
$$

where $r$ is the distance between particle centers. Force, $F_{\mathrm{LJ}}$, is the negative derivative of potential energy with respect to position, hence

$$
F_{\mathrm{LJ}}(r)=\frac{24}{r} \varepsilon\left[2\left(\frac{\sigma}{r}\right)^{12}-\left(\frac{\sigma}{r}\right)^{6}\right]
$$

The LJ/SF interaction, however, includes one more parameter which is cut-off distance $r_{c}$ : 


$$
U_{\mathrm{LJ} / \mathrm{SF}}(r)=\left\{\begin{array}{cc}
U_{\mathrm{LJ}}(r)-U_{\mathrm{LJ}}\left(r_{c}\right)+\left(r-r_{c}\right) F_{\mathrm{LJ}}\left(r_{c}\right) & r \leq r_{c} \\
0 & r>r_{c}
\end{array}\right.
$$

This formulation ensures a smooth energy/force transition at the cut-off distance. Parameters for interaction between dissimilar particles like active-CBD and active-active are calculated using standard combining rules:

$$
\sigma_{i j}=\frac{1}{2}\left(\sigma_{i i}+\sigma_{j j}\right), \varepsilon_{i j}=\left(\varepsilon_{i i} \varepsilon_{j j}\right)^{\frac{1}{2}}, r_{c, i j}=\frac{1}{2}\left(r_{c, i i}+r_{c, j j}\right)
$$

where indices $i$ and $j$ represent particle identities.

The LJ/SF potential is a conservative potential that does not contain all needed physics for mesoscale particle motion. Thus, the DPP2 model also uses the GH force field with its associated adjustable parameters. GH consists of a repulsive force in the normal direction, frictional contact in the tangential direction, and velocity-dependent dissipative forces that imitate solvent behavior, as discussed in more detail in the LAMMPS documentation [43].

"Best Place for Figure 1"

Figure 1 illustrates the general behavior of the LJ/SF and GH interactions. It shows the liquid slurry and solid normal-force interaction energy between two CBD particles, which in both cases is a linear combination of both $\mathrm{LJ} / \mathrm{SF}$ and GH types. It was found that the GH interaction generated results more characteristic of a liquid and the LJ/SF interaction generated results more characteristic of a solid due to the strong attraction needed to hold particles in a stable configuration. Therefore, the liquid potential is mostly GH and the solid potential is mostly $\mathrm{LJ} / \mathrm{SF}$. In order to do the comparison in Figure 1, the rotation- and velocity-dependent $\mathrm{GH}$ forces are set to zero, leaving the $\mathrm{GH}$ normal force that provides only repulsion. That normal force can be analytically integrated to yield a potential curve as 
shown in Figure 1.

\subsection{Carbon model}

Because of the large number of adjustable parameters in the force interactions between CBD and active particles, this can make determination of individual parameters difficult. Therefore a scheme was used to enable parametrization of the CBD potential from multiple experiments. Carbon simulations and experiments, free of active material particles, were first developed and used. Subsequently, active particles were added to the simulation and to the mixture used in experiments for additional parameter determination. As discussed below, all CBD particles in the system are considered to be identical.

One of the objectives of this work is to simulate a volume representative of real electrode films. For the commercial-grade electrodes we have in mind, this means a simulation on the order of $40 \mu \mathrm{m} \times$ $40 \mu \mathrm{m} \times 40 \mu \mathrm{m}$. At that size it would be computationally impractical to consider every single carbon black particle (around $50 \mathrm{~nm}$ diameter), just as it would be impractical to consider binder and solvent molecules individually. Along with the experimental observation that carbon and binder tend to form distinct aggregates in the solid, this justified the use of a CBD particle in the DPP2 model. One CBD particle represents approximately 1000 carbon black particles and associated binder. In the liquid state the CBD particle also includes an associated mass of solvent. Therefore during the drying process the mass and other characteristics of the CBD particle change. In summary, the CBD particle is meant to represent the aggregate cohesion, adhesion, and friction of its constituents while treating them in a coarse-grained homogenous fashion.

Nanoporosity in the dried carbon-binder domains (see Fig. $8 \mathrm{c}$ and e) highly affects mechanical properties of the electrode. In the simulation only macropores are explicitly treated, while CBD particles implicitly include nanoporosity. To imitate the mechanical effect of such nanoporosity in the 
dried state, CBD particles are made fairly soft compared to active particles (by choosing very soft potential functions). This homogenization of the nanoporosity would likewise affect electrochemical properties of CBD particles, such as their effective ionic and electronic conductivities, though such calculations are not included in this work.

The size of the CBD particles is not determined a priori. It involves tradeoffs between computational cost, spatial resolution of the structure, and resulting liquid and solid properties. We determined that an effective size on the order of $1 \mu \mathrm{m}$ was appropriate. As shown in Figure 1, the interactions between CBD particles are "soft" meaning the particles are intended to mutually overlap, especially in the liquid state. While there is not a fixed volume or diameter of the CBD particles, they do have diameter-like parameters in the granular and LJ/SF potentials, namely $d_{\mathrm{c}}$ and $\sigma_{\mathrm{c}}$. In the liquid state $d_{\mathrm{c}, 1}=6.2 \mu \mathrm{m}$ was selected primarily by matching simulation and experimental viscosities. Even though this might seem like a large diameter, the significant number of overlaps between particles means that the effective volume of the particle is greatly reduced.

A key idea in the DPP2 model is that the drying transition happens by changing some of the parameters in the LJ/SF and GH potentials, while holding other parameters constant. Specifically for the CBD particle there is one potential (force field) for liquid and another for solid. During drying the solvent component of the CBD particle is lost and partially gets replaced with nanopores (later to be filled with electrolyte). The loss of solvent allows the binder to better adhere particles to each other. This is modeled by reducing the granular diameter from $6.2 \mu \mathrm{m}$ to $d_{\mathrm{c}, \mathrm{s}}=1.3 \mu \mathrm{m}$ while holding $\sigma$ constant (see Table 1). Furthermore, energetic and friction parameters $\left(\varepsilon, K_{\mathrm{n}}, K_{\mathrm{t}}\right.$, and $\left.\mathrm{X}_{\mu}\right)$ are increased. The resulting CBD solid potential (see Figure 1) is more attractive than is the liquid potential. The changed potential reduces the size and increases the rigidity of the solid structure relative to the liquid. (The resulting effect of this drying transition is illustrated in Figure 4 below.) 
An iterative process was used to adjust liquid and solid CBD parameters in order to match experimental mechanical and structural properties in an average sense. Figure 2 is an example of the sensitivity analyses that were used to perform this parameterization process. In this case a series of simulations was done, each simulation having a different granular diameter $d_{c}$ while holding other parameters constant including final box volume. The figure shows the substantial effects on cell pressure, elasticity, and viscosity for the reduction in $d_{\mathrm{c}}$, meant to imitate the drying process. The properties are especially sensitive to $d_{c}$ because it controls the basic particle volume and (in combination with the LJ/SF potential) the attraction between particles. For instance, a negative pressure (tension) is generated for smaller $d_{\mathrm{c}}$ values, due to the fixed size of the box and increased interparticle attraction (Figure 2a). Similarly, the elasticity and viscosity increase greatly (Figure 2b and c), as one would expect when the slurry is dried. One also notices that as $d_{\mathrm{c}}$ is decreased that the uncertainty in properties increases, due to increased structural rigidity and relative lack of relaxation of the structure on the timescale sampled by these simulations. The interparticle parameters besides $d_{\mathrm{c}}$ that are changed during drying have more subtle effects and were viewed as a way to fine-tune the simulation behavior

With the loss of solvent mass during drying, the mass of the CBD particle changes as well. In LAMMPS the mass of granular-based particles is specified by a particle density in combination with the granular diameter. In this case the CBD particle densities (see Table 1) were picked so that the overall simulation can match experimental liquid and solid densities. When one compares the density of liquid CBD particles $\left(0.105 \mathrm{~g} \mathrm{~cm}^{-3}\right)$ to the overall slurry density $\left(1.03 \mathrm{~g} \mathrm{~cm}^{-3}\right)$, there is an obvious large degree of overlapping between adjacent particles. The level of particle overlap is lower in the dried film, but cannot be ignored since the additives tend to form aggregates as revealed by SEM (see Figure 8e and f). 


\section{"Best Place for Figure2"}

\subsection{Active model}

The active model was developed by adding the appropriate number and size of active particles into the carbon model to create a simulation with composition typical of a commercial film. Obviously the ratio of active material, additives, and solvent has a large effect on the behavior of the slurry and resulting dried film, and therefore this is a key property the simulation must match. The relative number of CBD and active particles were determined according to the composition of the slurry tested in the experiments, the density of the materials, and the distribution of active material particle sizes. To begin, the total number of active particles was assumed to be 53 for the baseline case. As discussed in the experimental section, the experimental active particle-size distribution (Figure 6c) then allows us to determine the number of particles for each of seven discrete sizes to represent that distribution. Next, we determine the total mass of active material (e.g. $6.99 \times 10^{-8}$ g) from the crystal density of active material (NCM-523, $\mathrm{LiNi}_{0.5} \mathrm{Co}_{0.2} \mathrm{Mn}_{0.3} \mathrm{O}_{2}$ ), which was calculated to be $4.789 \mathrm{~g} \mathrm{~cm}^{-3}$ [44]. The number of $\mathrm{CBD}$ particles is then determined from the experimental dry mass ratio of carbon and binder to active material, which is 0.111 as discussed in Section 3.1, as well as the dried CBD granular density $\left(0.93 \mathrm{~g} \mathrm{~cm}^{-3}\right)$ and size $(1.3 \mu \mathrm{m})$, obtained from the carbon model. This results in a total number of 7260 CBD particles in the baseline case.

Another issue regarding the active model was the shape of active particles. It has been proven that the shape and size of active particles has an important bearing on the microstructure and performance of lithium-ion batteries $[\underline{15}, \underline{17}, \underline{45-47]}$. In the samples we examined, the active material particles are generally spheroidal (see Figure 6), and so we treat the simulated active particles as spheres. Any roughness on the surface of the active particles is computed in the granular model with tangential friction terms. 
The parameters used for the active model (as well as the carbon model) are given in Table 1. Three principles were used to guide the parameterization of the active particle interactions. First, compared to the CBD particles, active particles are considered harder. To represent this property a stiffer potential is favored. However, this must be balanced against numerical stability and sampling efficiency, because stiffer potentials require shorter time steps in the simulation. Therefore some degree of overlap is allowed between active particles. A second physically motivated principle is that, in contrast to the carbon model, there should be minimal change to the active potential parameters between the liquid and solid states. It turns out that under the GH model available in the publicly-available and standard configuration of LAMMPS, only one set of $K_{\mathrm{n}}, K_{\mathrm{t}}, X_{\mu}, \gamma_{\mathrm{n}}$, and $\gamma_{\mathrm{t}}$ values may be used in a single simulation; therefore the active model must use the previously derived values of these parameters for the carbon model. Lastly, because there is a distribution of active particle sizes leading to a large number of the remaining adjustable parameters $\left(d_{\mathrm{a}}, \varepsilon_{\mathrm{a}}, \sigma_{\mathrm{a}}, r_{\mathrm{c}, \mathrm{a}}\right)$ for each size, simple relationships are designed to compute these interaction parameters as a function of each particle size, $d_{\mathrm{a} \text { exp }}$, determined from experiment. Cross-interactions between different particle types (e.g. CBD-active) or between different particle sizes (e.g. active-active) are computed according to the combining rules in Eq. (4).

\section{"Best place for table 1"}

The potential for the active particles is dominated by the LJ/SF potential, meaning that parameters $\varepsilon_{\mathrm{a}}, \sigma_{\mathrm{a}}$, and $r_{\mathrm{c}, \mathrm{a}}$ are critical and the $\mathrm{GH}$ parameters are less influential, regardless of the state of the simulation (liquid or solid). In particular, the interplay between the LJ/SF parameters allows one to control the repulsive (shorter range) and attractive (longer range) parts independently. As a general principle, elasticity and viscosity both increase with increased magnitude of potential but to differing degrees; subtle differences can be exploited to try to match these and other properties by adjusting the model parameters. Therefore to determine the parameters in Table 1, a sensitivity analysis similar to 
that shown in Figure 2 was used for the active particles, while holding the CBD parameters constant. For simplicity and computational efficiency, $d_{\mathrm{a}}$ and $r_{\mathrm{c}, \mathrm{a}}$ were set equal to each other.

\subsection{LAMMPS simulation details}

LAMMPS is generally used for molecular dynamic simulations, but in this work mesoscale particle sizes and times are used to explore the configuration space dynamically. Four types of simulations were run for both the carbon and active models: equilibration to liquid density, viscosity test of liquid, drying of liquid, and elasticity test of solid. Custom programs were also used for pre- and postprocessing. Details on simulation parameters are given below.

For computational cost-efficiency, periodic boundary conditions were utilized. This means that a relatively small-size simulation can imitate bulk slurry and bulk film behavior.

\subsubsection{Slurry equilibration}

Both carbon and active models were started by generating a well-mixed liquid representing the slurry. For the carbon model, 5500 CBD particles were randomly placed in a cubic box of size $900 \mu \mathrm{m}$ while rejecting positions with particle overlap. The large box size makes the zero-overlap possible. Particles were assigned initial random velocities corresponding to the temperature, $300 \mathrm{~K}$. In this condition the pressure of the system was close to zero since there is no interaction between particles. In order to get to the fabrication process conditions, the simulation was run under NPT control at 1 bar pressure, causing the box to shrink until a stable liquid-like density is achieved. The final size of the cubic box was around $41.3 \mu \mathrm{m}$. The simulation required $7.1 \times 10^{6}$ time steps of size $\Delta t=0.001 \mu \mathrm{s}$.

The equilibration for the active model was performed nearly identically to that for the carbon model. The final size of the cubic box containing 53 active particles and 7260 CBD particles was approximately $46.4 \mu \mathrm{m}$. The equilibration took approximately 6 to 8 hours with 24 processors on a 
shared supercomputer resource. The resulting configuration was the initial point for drying and viscosity computations (Figure 3a).

For visualization purposes and to ensure model independency from simulation size, carbon and active simulations were repeated three times with double the number of particles in a box elongated in the $z$ direction (the direction of shrinkage during drying) so that dried thickness in that direction better matched experimental film thicknesses. This resulted in equilibrated liquid slurry boxes of size 32.3 $\mu \mathrm{m} \times 32.3 \mu \mathrm{m} \times 130.8 \mu \mathrm{m}$ for the carbon model and $37.2 \mu \mathrm{m} \times 37.2 \mu \mathrm{m} \times 148.8 \mu \mathrm{m}$ for the active model. Note that the illustrations in Figure 3 and subsequent figures show results from this set of elongatedbox simulations. However, no significant difference in predicted physical properties was observed between runs initiated with elongated vs. cubic boxes.

\subsubsection{Viscosity computation}

The viscosity of the slurry in both carbon and active simulation was computed by means of an algorithm known as non-equilibrium MD (NEMD), in which the simulation box is deformed. The normal periodic boundaries are modified to become what are known as Lee-Edwards boundary conditions [48-50]. This method has been used routinely for evaluating the viscosity of different fluids. As shown in Figure $3 \mathrm{~b}$, the simulated box was sheared in the $x$ direction at a specified rate. The viscosity is the ratio between the computed shear stress and the shear rate. The average shear stress was computed according to the velocities and forces between particles. For illustration, Video 1 shows how the simulation box is sheared in NEMD method. A comparison between simulation and experiment is given in Section 4.1 and Figure 7.

"Best place for Figure 3" 


\subsubsection{Drying}

In this work the drying process for both the carbon and active models is approximated as follows. As discussed in the previous sections, in transitioning from liquid to solid models, the attraction between particles increases and the size of the CBD particles decreases considerably. This replicates the effect of loss of solvent. The change in particle parameters immediately causes a negative stress in the box in all directions (see Figure 2a). The drying process is imitated by next allowing the box to shrink in the $z$ direction, while holding constant the dimensions in the $x$ and $y$ directions. This 1-D shrinking process, shown in Figure 4, continues until the stress in the $z$ direction equilibrates to 1 bar (using NPT control). This equilibrium was found to require $1.61 \times 10^{7}$ time steps, with the same $\Delta \mathrm{t}$ as the previous steps, to get to a reasonable level of equilibration. This exceeds the number of time steps required for the earlier liquid equilibration, likely because particle motion is hindered in the solid state relative to the liquid state. Video 2 is basically a sequence of LAMMPS simulation images (outputs of consecutive time steps) during the drying process. This video clearly shows that CBD particles shrink and adhere together as well as to active particles. It also shows the electrode shrinks in $z$ direction and macropores form.

Just as in the real system, the solid stresses in the $x$ and $y$ directions do not equilibrate to atmospheric pressure because shrinkage is prevented in the plane of the electrode film. This is observed experimentally when, due to this negative in-plane stress, occasional cracks form (see, for instance, Figure 8e and f) and also the edges of a film dried on a current collector have a tendency to curl upward. (This is mechanically similar to how a bimetallic strip curls when the temperature is changed.) The ratio of box length in the $z$ direction before and after drying is known as the shrink ratio and is a key metric we compare between model and experiment below.

"Best place for Figure 4" 


\subsubsection{Elasticity test}

The final type of LAMMPS simulation is to test the elasticity or stiffness of the solid. Although there are several moduli for elasticity evaluation, Young's modulus was used in this work in both LAMMPS simulations and experiments. Young's modulus of the dried film was computed by incrementally and sequentially deforming the simulation box in the $x, y$, and $z$ directions. The elasticity is obtained from the ratio of stress change to strain (see experimental section below).

\section{Experiments}

\subsection{Electrode fabrication}

The required slurries and dried films were made as follows. The materials, compositions, and fabrication steps were designed to imitate Toda 523 cathodes made by Argonne National Laboratory and used in our previous work [51]. The carbon slurry first was made by mixing carbon black (TIMCAL Super C45) with $15.66 \mathrm{~g}$ binder solution that contained 92 wt\% NMP solvent (Sigma Aldrich) and $8 \mathrm{wt} \%$ polyvinylidene fluoride (PVDF, Solvay Solef 5130) binder to maintain a carbonbinder dry weight ratio of 50:50. Likewise, the active slurry was prepared by adding $22.5 \mathrm{~g}$ active material (Toda NCM-523) to the above amounts. Therefore, the dry weight composition of active slurry is $90 \mathrm{wt} \%$ NCM-523, $5 \mathrm{wt} \%$ carbon black, and $5 \mathrm{wt} \%$ binder.

The fabrication process started by mixing the binder and solvent together at $80^{\circ} \mathrm{C}$ for 20 minutes in a closed vessel to make the binder solution. For carbon slurries the carbon black was added to the prepared solution and was stirred by an ultrasonic homogenizer for 15 minutes. For the active slurry, dry active material and carbon black were mixed well and ground by hand with a mortar and pestle. This powder mixture was then added to the binder solution and stirred by an ultrasonic homogenizer for 15 minutes. 
To make a solid film, the corresponding slurry was poured over a smoothed piece of aluminum foil (current collector) and thinned to the final coating thickness by use of a doctor blade. Controlling and knowing the thickness of the wet film is important for determining the shrinkage ratio. To have a uniform and smooth coating we performed the coating process in multiple stages from a thicker coat to the thickness of interest $(200 \mu \mathrm{m})$ by decreasing the height of the doctor blade and passing over the sample at an approximate rate of $1 \mathrm{~cm} / \mathrm{s}$. Finally, the wet electrodes were placed in a vacuum oven for 30 minutes. The thickness of wet film for both carbon and active films (excluding the aluminum current collector) was $177 \mu \mathrm{m}$ As a result of the drying process, carbon and active films shrank to 20.5 $\mu \mathrm{m}$ and $53.9 \mu \mathrm{m}$ average thickness, respectively, with averages determined by micrometer measurements at multiple locations. The experimental shrinkage ratio and other properties of the slurries and dried films are given in Table 2.

\subsection{Viscosity and elasticity measurements}

Part of the prepared slurry was used for the viscosity tests. An AR2000EX Rheometer (TA Instruments, New Castle, DE) was used to measure viscosity over a range of shear rates considered relevant to electrode fabrication, namely values between $1 \mathrm{~s}^{-1}$ and $1100 \mathrm{~s}^{-1}$ (see Figure 7). Temperature was controlled at $300 \mathrm{~K}$.

Conducting elasticity tests was found to be more challenging. The first step was delamination of the electrodes $[\underline{3}, \underline{8}, \underline{52}]$. In this work liquid gallium was used to dissolve the aluminum current collector as described by Zacharias et al. [8] The delaminated films are particularly fragile and thin, so standard elasticity measurement tools are not satisfactory. Therefore we devised an apparatus better suited to this measurement.

The elasticity apparatus contained a force sensor (FlexiForce Sensor-25lb, Tekscan, South Boston, MA), a micrometer (Chicago brand P.N 50059), a digital multimeter (Agilent 34410A), and a generic 
clamp as shown in Figure 5a. Before taking the tests, the force sensor was calibrated as instructed by the manufacturer and the resistance of the probe was shown to be linearly related to the applied force. We also calibrated the Young's modulus of the force probe itself; this was found to be much higher than for the samples and so strain in the force probe could be neglected in subsequent calculations. Stress was calculated by dividing the amount of force at each increment by the micrometer anvil surface area $\left(23.27 \times 10^{-5} \mathrm{~m}^{2}\right)$. Strain was calculated by the change of thickness divided by initial thickness of sample film(s).

The initial part of stress-strain graph is, theoretically, supposed to be linear, with the slope being the Young's modulus. Figure $5 \mathrm{~b}$ is the stress-strain graph obtained from 4 different tests for each sample. The slope (elasticity) and its uncertainty are reported in Table 2.

"Best place for Figure 5"

\subsection{Imaging and segmentation}

In order to compare the simulated microstructure to the real microstructure, a scanning electron microscope with focused ion beam (SEM/FIB, FEI Helios Nanolab 600) was employed to produce a few representative 2D cross sections (e.g. Figure 6a, Figure 8c). One of these was then segmented to produce the three domain types (Figure 8d). The segmentation was performed manually by use of photo imaging software. It is important to distinguish between nanopores and macropores while segmenting. In this work pores smaller in diameter than $d_{\mathrm{c}}(1.3 \mu \mathrm{m})$ were treated as part of CBD domains to be consistent with the simulations.

Another purpose of using SEM/FIB images was to obtain a size distribution of active particles for use in the active simulation, as discussed in Section 2.4. An experimental particle-size distribution was collected by measuring 249 active material particles ranging from 0.8 to $15 \mu \mathrm{m}$ that could be visually 
isolated from 85 sequential SEM/FIB image slices from a calendered Toda 523 cathode sample. Each slice was separated by $0.5 \mu \mathrm{m}$. The size of each active material particle was obtained by first finding the $2 \mathrm{D}$ image where the particle's cross sectional area was greatest. For that image the two longestpossible perpendicular lines or chords were drawn (See Figure 6a). A geometric average of these two line lengths was taken to be the effective diameter for the particle. The resulting smoothed distribution of diameters is shown in Figure 6c.

\section{"Best Place for Figure 6"}

For convenience and efficiency in the LAMMPS simulation, it is preferable to have a relatively small number of discrete particle sizes, as discussed above. Therefore the smoothed particle-size distribution was re-divided into 7 bins with approximately $2-\mu \mathrm{m}$ size increments. The effective or representative diameter for each bin was chosen to as the root-mean-square diameter for all particles in the bin. This allows the discrete model to conserve particle surface area, a key property for electrochemical reaction modeling (though reactions are not modeled in this work). The resulting discrete particle sizes are $d_{\mathrm{a} \text { exp }}=2.15,4.21,6.17,8.07,9.95,11.56$, and $15.26 \mu \mathrm{m}$; the corresponding particle numbers are $10,6,10,14,8,4$, and 1 , respectively.

Later in the work, and as a validation step for the size distribution, an SEM image of active material powder was taken as shown in Figure 6b. This SEM image, which coincidentally contains 53 particles, confirms that active particles are spheroidal and gives a size distribution consistent with Figure 6c.

\section{Results and discussion}

Here we compare simulation and experimental results, to determine how well the model reproduces 
structural and mechanical properties. In this work parameterization and validation of the model cannot be cleanly separated. Even though many of the properties discussed in this section were considered in the parameterization steps, they can also be considered part of the validation steps. This is because the model is of a "first principles" type in which interparticle force parameters do not directly relate to desired physical properties. Therefore agreement between model and experiment is not guaranteed even with careful parameterization.

The error bars in Figure 7 and Table 2 are 95\% confidence intervals, which for simulations come from independent runs with different starting points. Relatively small error bars demonstrate that the simulations are independent of the starting points, and the simulation time and size are sufficiently large.

\subsection{Viscosity}

The shear viscosity of a fluid is a measure of its resistance to deformation by shearing motion and is an important parameter controlling the coating process. It has been shown that the slurry used for Liion batteries exhibits substantial shear thinning (i.e. non-Newtonian behavior) [53-55], as also observed here by simulation and experiment.

Viscosity was chosen as a key metric for parameterization and validation of the carbon and active slurry models. As seen in Figure 7 the carbon and active models correctly predict the shear-thinning behavior of the slurries. As discussed in the previous sections, an iterative method was followed by modifying the simulation parameters according to the sensitivity of the system to the parameters (see Figure 2). It was observed that increased $\gamma_{\mathrm{n}}$ and $\gamma_{\mathrm{t}}$ values tended to increase viscosity, more especially at the higher shear rates, causing the viscosity to have less dependence on shear rate. Likewise, viscosity increased with some parameters like $K_{\mathrm{n}}, K_{\mathrm{t}}, \varepsilon, \sigma$. However, the density of particles did not have a considerable effect on viscosity. Larger values of $X_{\mu}$ and smaller values of granular diameter $d_{\mathrm{c}}$ 
each tended to increase viscosity and, notably, the variability in viscosity results. Therefore, for the liquid model $X_{\mu}$ was kept small and $d_{\mathrm{c}}$ was kept large, as given in Table 1 .

\section{"Best Place for Figure 7"}

Figure 7 indicates how viscosity results from simulation (symbols with error bars) are in quantitative agreement with experimental viscosity (lines) for the shear rates used in the coating process. Manufacturers of Li-ion batteries are, recently, more interested in higher coating speed (shear rates greater than $500 \mathrm{~s}^{-1}$ ) where our simulation shows consistent agreement with experiment. Our experimental results are quite similar to the rheology reported by Bauer and Notzel for a slurry similar to that used here [56].

\subsection{Other properties}

Table 2 compares additional properties predicted by the carbon and active models with the corresponding experiments. As discussed above, elasticity in the $z$ direction was used for parameterization of the solid carbon and active models because it is a key physical parameter and can be rapidly computed and measured. As was done for viscosity, several parameters were adjusted to understand their effects on the predicted elasticity. It was observed that $\varepsilon, K_{\mathrm{n}}$, and $K_{\mathrm{t}}$ each had a strong effect on the elasticity, with increased parameter values leading to higher elasticity. $K_{\mathrm{n}}$, and $K_{\mathrm{t}}$ were determined for the solid carbon model and not subsequently changed for the active model. As seen in Table 2, elasticity results from simulations are in good agreement with experimental elasticities.

\section{"Best place for table 2"}

Shrinkage ratio is an important factor in the drying process. A higher degree of shrinkage provides a lower amount of pores and cracks in the dried electrode. The experimental shrinkage ratio was simply calculated by measuring the thickness of the wet and dried films (not including current collector), 
while for simulations the $z$-length of the simulation box before and after drying gives the shrinkage ratio. As seen in Table 2 the shrinkage ratio of the carbon model is $7 \%$ lower and the active model is $19 \%$ lower than the corresponding experiments. This is reasonable agreement, given that the drying procedure in the model is more rapid than in the experiments. One would expect that different drying procedures could yield different shrinkage ratios as well as different resulting morphologies.

Liquid density and film density were calculated according to the particle sizes, densities, and numbers, as well as the size of the simulation box. The simulation results in Table 2 show considerable agreement with experiment. As discussed in Section 2.3, there is considerable overlap between CBD particles in this simulation. The simulation errors come from different simulation box sizes in different runs (each simulation equilibrates to the same pressure, but has a slightly different volume). Experimental liquid densities do not have error ranges - the experiments were not repeated-but are expected to have quite low uncertainty due to the volumes and masses of slurry used.

\subsection{Structure determination}

When the electrode coating is dried, volume or thickness decreases and the attraction increases significantly between carbon, binder, and active material, due to loss of solvent. This leads to the generation of macropores or cracks, as shown in Figure 8e and $\mathrm{f}$. In addition, carbon and binder agglomerate and adhere to the active material. These microstructural features must be reproduced by the model if it is to be considered accurate.

In order to compare the microstructure from the LAMMPS simulation to SEM/FIB experiment, the final configuration from LAMMPS must be segmented as was the experiment (see Section 3.3). This was done with a customized algorithm as follows. First the coordinates of the center of each particle were obtained from the LAMMPS configuration file. The simulation volume was divided into cubic voxels with length $0.5 \mu \mathrm{m}$, the same size as used experimentally. Each voxel was assigned as active if 
it was within distance $0.5 d_{\mathrm{a} \text { exp }}$ from the nearest active particle center. If not active, the voxel is tested as $\mathrm{CBD}$ if it is within $0.5 d_{\mathrm{c}}$ eff from the nearest CBD particle center. If neither of these conditions is met, then the voxel is assigned as pore. Because there is no defined experimental value of CBD size (in contrast to active material), $d_{\mathrm{c} \text { eff }}$ was empirically adjusted to be $1.5 d_{\mathrm{c}}$, or somewhat larger than the granular diameter. The criterion for adjustment was to approximately match the experimental ratio of CBD to pore volumes.

Finally, when the simulated structure has been turned into a grid occupied by the 3 phases, one can examine overall volume fractions (Table 2) or visualize it in the same manner as SEM/FIB images (Figure 8).

For the carbon model and experiment, the volume fractions of CBD and macro-pore match well between simulation and experiment, as expected. As for the active model and experiment, there is a modest mismatch for volume fractions. This could be caused by limited experimental sampling (only one $2 \mathrm{D}$ cross section was used), and by the fact that the simulation exhibits less shrinkage than the experiment. Nevertheless, the volume fractions between simulation and experiment are reasonably consistent.

\section{"Best Place for Figure 8"}

Figure 8 reveals the close resemblance between a representative cross-section of the simulated dried film (part b) and an SEM/FIM image of the real electrode (parts c and d), particularly the qualitative shape of the pore and carbon mains. Figure 8 also reveals the evolution of the structure from the liquid (part a) to solid (part b) states, for which we do not yet have experimental comparison. As expected, macropores are not present in the liquid slurry, and form upon drying due to the increased attraction and reduction in size of the CBD particles. Similarly, the carbon-binder aggregates are attracted to the active particles, forming an electronically conducting bridge between them. 


\section{Conclusion}

In this work a novel mesoscale particle-based simulation technique has been developed to predict the microstructure of Li-ion battery electrodes produced by a traditional fabrication process. The structures before (slurry) and after (solid film) drying were predicted. The dried film structure was shown to be in acceptable agreement with the structure of the real electrode. There is also a reasonable match between simulation and experiment for other physical and mechanical properties.

To make the simulation computationally feasible for this size of simulation (around100 $\mu \mathrm{m}$ ), carbon black particles (around $50 \mathrm{~nm}$ diameter) and polymeric binder molecules are lumped together to form CBD particles with size around 1-6 $\mu \mathrm{m}$. The CBD particles also include solvent in the case of liquid simulations and nanopores in the case of solid simulations. This permits a simulation with only two types of particles: CBD and active, though multiple sizes of active particles were also used.

Two kinds of potential functions were used to describe particle interactions: shifted-force LennardJones (LJ/SF) and granular Hertzian (GH). Since many parameters needed to be regressed, this was performed in two sequential steps by means of a carbon-only model and a model with the full electrode composition.

In order to know if a particle-based simulation is reproducing the real system accurately, we chose a number of easily measured experimental properties like viscosity, shrinkage ratio during drying, elasticity, and volume fraction of phases, to compare to the simulation results.

Viscosity of the slurry was chosen because it plays a pivotal role in the coating process. For instance, it is difficult to achieve a smooth and uniform coating if the viscosity is too high. While viscosity can be lowered by adding additional solvent, this is undesirable because it increases porosity of the dried film. In our simulations and experiments, highly shear-thinning behavior of a Li-ion slurry 
was shown clearly. These results suggest that coating electrodes at higher shear rates may improve the structure of electrodes or reduce the amount of solvent needed, in addition to the inherent economic benefit of a faster coating process. Additional work is needed to determine if this is in fact the case.

We introduced in this work a drying metric known as the shrinkage ratio. We are not aware of this being used previously in published work in the battery field. This property can be used in addition to porosity and loading of the electrode to characterize the fabrication process by manufacturers. High shrinkage ratio corresponds to a high degree of particle rearrangement during drying. Different drying mechanisms may affect the shrinkage ratio and likewise the arrangement of particles and electrochemical properties. Again, this is a topic that deserves further study.

Elasticity of the dried film (and the stress-strain curve in general) was used in this work as an indicator of the mechanical stability of the dried film which is very important in the calendering process, though calendering was not covered in the present work. Stress-strain properties of the precalendered film have received little attention as manufacturing metrics, and will be examined more closely in our future work.

Future work planned by our group will expand the applicability of the particle model by applying it to other types of active material (e.g. non-spheroidal shapes) and binder. The use of periodic boundaries in all three directions will be relaxed, to enable the generation of air/liquid and air/solid interfaces necessary to study the shape of the interface as a result of different fabrication steps, including calendering. Furthermore, the structures produced by the model can be analyzed for effective transport parameters (i.e. electronic conductivity, ionic conductivity, and diffusivity), which can be compared to experiment. Our long-term goal is to integrate the particle model with a multiscale electrochemical model that will enable optimization of the fabrication process for specific battery performance metrics. 


\section{Acknowledgements}

This work was supported by the BMR program of the U.S. Department of Energy. The authors would like to thank Bryant Polzin for providing electrode materials.

\section{References}

[1] P. Shearing, R. Bradley, J. Gelb, N. Brandon, P. Withers, Microsc Microanal 17 (2011) 1672-1673.

[2] D.R. Nevers, S.W. Peterson, L. Robertson, C. Chubbuck, J. Flygare, K. Cole, D.R. Wheeler, J. Electrochem. Soc. 161 (2014) A1691-A1697.

[3] I.V. Thorat, D.E. Stephenson, N.A. Zacharias, K. Zaghib, J.N. Harb, D.R. Wheeler, J. Power Sources 188 (2009) 592-600.

[4] S.E. Trask, Y. Li, J.J. Kubal, M. Bettge, B.J. Polzin, Y. Zhu, A.N. Jansen, D.P. Abraham, J. Power Sources 259 (2014) 233-244.

[5] M.E. Spahr, D. Goers, A. Leone, S. Stallone, E. Grivei, J. Power Sources 196 (2011) 3404-3413.

[6] H. Zheng, R. Yang, G. Liu, X. Song, V.S. Battaglia, J. Phys. Chem. 116 (2012) 4875-4882.

[7] D.E. Stephenson, B.C. Walker, C.B. Skelton, E.P. Gorzkowski, D.J. Rowenhorst, D.R. Wheeler, J. Electrochem. Soc. 158 (2011) A781-A789.

[8] N.A. Zacharias, D.R. Nevers, C. Skelton, K. Knackstedt, D.E. Stephenson, D.R. Wheeler, J. Electrochem. Soc. 160 (2013) A306-A311.

[9] L. Zielke, T. Hutzenlaub, D.R. Wheeler, I. Manke, T. Arlt, N. Paust, R. Zengerle, S. Thiele, Adv. Energy Mater. 4 (2014) 1301617.

[10] L. Zielke, T. Hutzenlaub, D.R. Wheeler, C.-W. Chao, I. Manke, A. Hilger, N. Paust, R. Zengerle, S. Thiele, Adv. Energy Mater. 5 (2014) 1401612.

[11] Z. Liu, V. Battaglia, P.P. Mukherjee, Langmuir 30 (2014) 15102-15113.

[12] J. Li, C. Daniel, D.L. Wood, Cathode Manufacturing for Lithium-Ion Batteries, in: Handbook of 
Battery Materials, Wiley-VCH Verlag GmbH \& Co. KGaA 2011, pp. 939-960.

[13] T. Marks, S. Trussler, A.J. Smith, D. Xiong, J.R. Dahn, J. Electrochem. Soc. 158 (2011) A51A57.

[14] B. Bitsch, J. Dittmann, M. Schmitt, P. Scharfer, W. Schabel, N. Willenbacher, J. Power Sources 265 (2014) 81-90.

[15] D.-W. Chung, P.R. Shearing, N.P. Brandon, S.J. Harris, R.E. García, J. Electrochem. Soc. 161 (2014) A422-A430.

[16] L.-C.C. Darjen Liu, Ta-Jo Liu, Tan Fan, Erh-Yeh Tsou, Carlos Tiu, Adv. Chem. Engineer. Sci. 4 (2014) 515-528.

[17] M.A. Martin, C.-F. Chen, P.P. Mukherjee, S. Pannala, J.-F. Dietiker, J.A. Turner, D. Ranjan, J. Electrochem. Soc. 162 (2015) A991-A1002.

[18] Z. Liu, P.P. Mukherjee, J. Electrochem. Soc. 161 (2014) E3248-E3258.

[19] J. Li, C. Daniel, D. Wood, J. Power Sources 196 (2011) 2452-2460.

[20] G. Liu, H. Zheng, S. Kim, Y. Deng, A.M. Minor, X. Song, V.S. Battaglia, J. Electrochem. Soc. 155 (2008) A887-A892.

[21] H.Y. Tran, G. Greco, C. Täubert, M. Wohlfahrt-Mehrens, W. Haselrieder, A. Kwade, J. Power Sources 210 (2012) 276-285.

[22] W. Haselrieder, S. Ivanov, D.K. Christen, H. Bockholt, A. Kwade, ECS Trans 50 (2013) 59-70.

[23] B. Westphal, H. Bockholt, T. Günther, W. Haselrieder, A. Kwade, ECS Trans 64 (2015) 57-68.

[24] W. Bauer, D. Nötzel, V. Wenzel, H. Nirschl, J. Power Sources 288 (2015) 359-367.

[25] P.R. Shearing, N.P. Brandon, J. Gelb, R. Bradley, P.J. Withers, A.J. Marquis, S. Cooper, S.J. Harris, J. Electrochem. Soc. 159 (2012) A1023-A1027.

[26] M. Ebner, F. Geldmacher, F. Marone, M. Stampanoni, V. Wood, Adv. Energy Mater. 3 (2013) 845-850. 
[27] M. Ebner, D.-W. Chung, R.E. García, V. Wood, Adv. Energy Mater. 4 (2014) 1301278.

[28] D.S. Eastwood, R.S. Bradley, F. Tariq, S.J. Cooper, O.O. Taiwo, J. Gelb, A. Merkle, D.J.L. Brett, N.P. Brandon, P.J. Withers, P.D. Lee, P.R. Shearing, Nucl Instrum Meth B 324 (2014) 118-123.

[29] M. Ender, J. Joos, T. Carraro, E. Ivers-Tiffée, J. Electrochem. Soc. 159 (2012) A972-A980.

[30] J.R. Wilson, J.S. Cronin, S.A. Barnett, S.J. Harris, J. Power Sources 196 (2011) 3443-3447.

[31] S.J. Cooper, D.S. Eastwood, J. Gelb, G. Damblanc, D.J.L. Brett, R.S. Bradley, P.J. Withers, P.D. Lee, A.J. Marquis, N.P. Brandon, P.R. Shearing, J. Power Sources 247 (2014) 1033-1039.

[32] Y.-c.K. Chen-Wiegart, P. Shearing, Q. Yuan, A. Tkachuk, J. Wang, Electrochem. Commun. 21 (2012) 58-61.

[33] S. Komini Babu, A.I. Mohamed, J.F. Whitacre, S. Litster, J. Power Sources, 283 (2015) 314-319.

[34] B. Yan, C. Lim, L. Yin, L. Zhu, J. Electrochem. Soc. 159 (2012) A1604-A1614.

[35] J. Joos, T. Carraro, M. Ender, B. Rüger, A. Weber, E. Ivers-Tiffée, ECS Trans 35 (2011) 23572368.

[36] T. Hutzenlaub, S. Thiele, R. Zengerle, C. Ziegler, Electrochem. Solid-State Lett. 15 (2011) A33A36.

[37] C. Wieser, T. Prill, K. Schladitz, J. Power Sources 277 (2015) 64-75.

[38] S. Vierrath, L. Zielke, R. Moroni, A. Mondon, D.R. Wheeler, R. Zengerle, S. Thiele, Electrochem. Commun. 60 (2015) 176-179.

[39] W. Wu, F. Jiang, Int. J. Hydrogen Energy 39 (2014) 15894-15906.

[40] R. Thiedmann, O. Stenzel, A. Spettl, P.R. Shearing, S.J. Harris, N.P. Brandon, V. Schmidt, Comp. Mat. Sci. 50 (2011) 3365-3376.

[41] S. Cheng, G.S. Grest, J. Chem. Phys. 138 (2013) 064701.

[42] M. Cerbelaud, B. Lestriez, A. Videcoq, R. Ferrando, D. Guyomard, J. Electrochem. Soc. 162 (2015) A1485-A1492. 
[43] granular Hertzian, lammps.sandia.gov (accessed July 2015).

[44] L. Xia, K. Qiu, Y. Gao, X. He, F. Zhou, J. Mater. Sci. 50 (2015) 2914-2920.

[45] S.P. Sheu, C.Y. Yao, J.M. Chen, Y.C. Chiou, J. Power Sources 68 (1997) 533-535.

[46] W. Alan, R.W. Stephen, P.P. Albert, Journal of Physics: Condensed Matter 19 (2007) 406215.

[47] H. Huang, C.H. Chen, R.C. Perego, E.M. Kelder, L. Chen, J. Schoonman, W.J. Weydanz, D.W. Nielsen, Solid State Ionics 127 (2000) 31-42.

[48] D.R. Wheeler, N.G. Fuller, R.L. Rowley, Mol. Phys. 92 (1997) 55-62.

[49] N.G. Fuller, R.L. Rowley, International Journal of Thermophysics 21 (2000) 45-55.

[50] Y. Yang, T.A. Pakkanen, R.L. Rowley, Int. J. Thermophys. 23 (2002) 1441-1454.

[51] B.J. Lanterman, A.A. Riet, N.S. Gates, J.D. Flygare, A.D. Cutler, J.E. Vogel, D.R. Wheeler, B.A. Mazzeo, J. Electrochem. Soc. 162 (2015) A2145-A2151.

[52] S.W. Peterson, D.R. Wheeler, J. Electrochem. Soc. 161 (2014) A2175-A2181.

[53] H. Zheng, L. Zhang, G. Liu, X. Song, V.S. Battaglia, J. Power Sources 217 (2012) 530-537.

[54] M. Yoo, C.W. Frank, S. Mori, Chem. Mater. 15 (2003) 850-861.

[55] J. Li, B.L. Armstrong, J. Kiggans, C. Daniel, D.L. Wood, Langmuir 28 (2012) 3783-3790.

[56] W. Bauer, D. Nötzel, Ceram. Int. 40 (2014) 4591-4598. 
Table 1: Interaction parameters of carbon binder domain (CBD) and active particles. $d_{\mathrm{a} \text { exp }}$ is assumed to be in units $\mu \mathrm{m}$.

\begin{tabular}{|c|c|c|c|c|c|c|}
\hline & Parameter & Unit & $\begin{array}{c}\text { CBD } \\
\text { Liquid }\end{array}$ & $\begin{array}{l}\text { CBD } \\
\text { Solid }\end{array}$ & $\begin{array}{l}\text { Active } \\
\text { Liquid }\end{array}$ & $\begin{array}{c}\text { Active } \\
\text { Solid } \\
\end{array}$ \\
\hline \multirow{3}{*}{$\mathrm{LJ}$} & $\varepsilon$ & $\operatorname{pg} \mu \mathrm{m}^{2} \mu \mathrm{s}^{-2}$ & 10 & 300 & $300\left(d_{\mathrm{a} \exp }\right)^{3}$ & $300\left(d_{\mathrm{a} \exp }\right)^{3}$ \\
\hline & $\sigma$ & $\mu \mathrm{m}$ & 1.1 & 1.1 & $0.88\left(d_{\mathrm{a} \exp }\right)$ & $0.88\left(d_{\mathrm{a} \exp }\right)$ \\
\hline & $r_{\mathrm{c}}$ & $\mu \mathrm{m}$ & 2.2 & 2.2 & $1.14\left(d_{\mathrm{a} \exp }\right)$ & $1.14\left(d_{\mathrm{a} \exp }\right)$ \\
\hline \multirow{7}{*}{$\mathrm{GH}$} & $d$ & $\mu \mathrm{m}$ & 6.2 & 1.3 & $1.14\left(d_{\mathrm{a} \exp }\right)$ & $1.14\left(d_{\mathrm{a} \exp }\right)$ \\
\hline & $K_{\mathrm{n}}$ & $\operatorname{pg} \mu \mathrm{m}^{-1} \mu \mathrm{s}^{-2}$ & 10 & 300 & 10 & 300 \\
\hline & $K_{\mathrm{t}}$ & $\operatorname{pg} \mu \mathrm{m}^{-1} \mu \mathrm{s}^{-2}$ & 13.6 & 408 & 13.6 & 408 \\
\hline & $\gamma_{\mathrm{n}}$ & $\mu \mathrm{m}^{-1} \mu \mathrm{s}^{-1}$ & 18 & 18 & 18 & 18 \\
\hline & $\gamma_{t}$ & $\mu \mathrm{m}^{-1} \mu \mathrm{s}^{-1}$ & 18 & 18 & 18 & 18 \\
\hline & $X_{\mu}$ & Unitless & 0.001 & 15 & 0.001 & 15 \\
\hline & $\rho_{p}$ & $\mathrm{pg} \mu \mathrm{m}^{-3}$ & 0.10 & 0.93 & 4.8 & 4.8 \\
\hline
\end{tabular}

Table 2: Comparison of key properties between simulation and experiment, with $95 \%$ confidence intervals where repeated independent samples were collected. Volume fractions are for the solid state.

\begin{tabular}{lcc|cc}
\hline & $\begin{array}{c}\text { Carbon } \\
\text { Simulation }\end{array}$ & $\begin{array}{c}\text { Carbon } \\
\text { Experiment }\end{array}$ & $\begin{array}{c}\text { Active } \\
\text { Simulation }\end{array}$ & $\begin{array}{c}\text { Active } \\
\text { Experiment }\end{array}$ \\
\hline Elasticity (MPa) & $9.55 \pm 0.15$ & $9.32 \pm 0.25$ & $11.10 \pm 0.73$ & $11.32 \pm 0.17$ \\
Shrinkage Ratio & $8.11 \pm 0.04$ & $8.70 \pm 0.59$ & $2.67 \pm 0.05$ & $3.29 \pm 0.08$ \\
Liquid Density $\left(\mathrm{g} \mathrm{cm}^{-3}\right)$ & $1.027 \pm 0.000$ & 1.03 & $1.66 \pm 0.00$ & 1.65 \\
Solid Density $\left(\mathrm{g} \mathrm{cm}^{-3}\right)$ & $0.681 \pm 0.004$ & $0.696 \pm 0.028$ & $2.070 \pm 0.037$ & $2.029 \pm 0.043$ \\
Active Volume Fraction & - & - & $0.41 \pm 0.03$ & 0.45 \\
CBD Volume Fraction & 0.90 & 0.89 & $0.40 \pm 0.01$ & 0.36 \\
Macro-pore Volume Fraction & 0.10 & 0.11 & $0.19 \pm 0.04$ & 0.19 \\
\hline
\end{tabular}




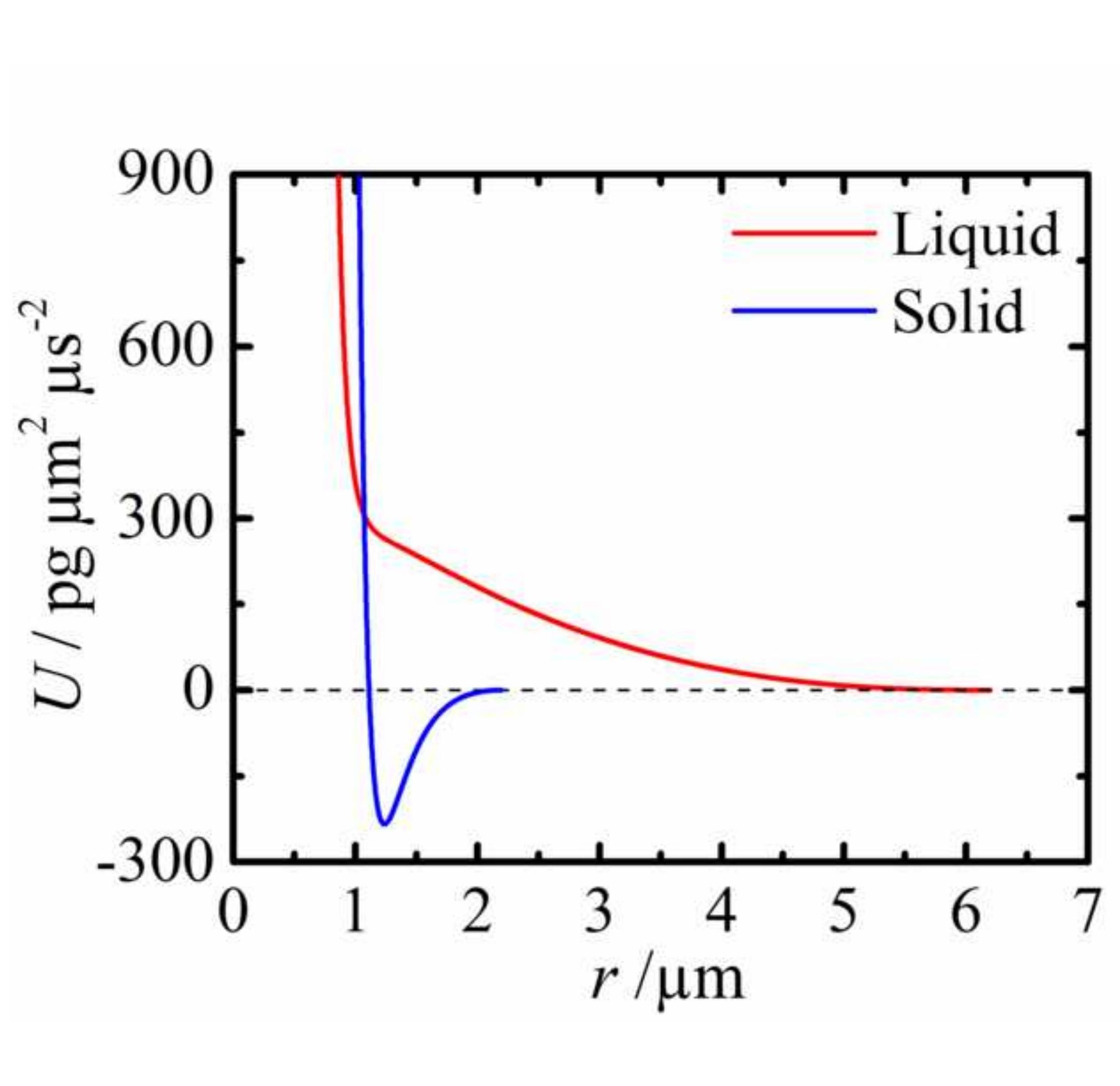

.

\author{
$r / \mu \mathrm{m}$
}

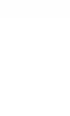

.

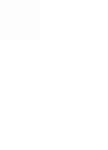
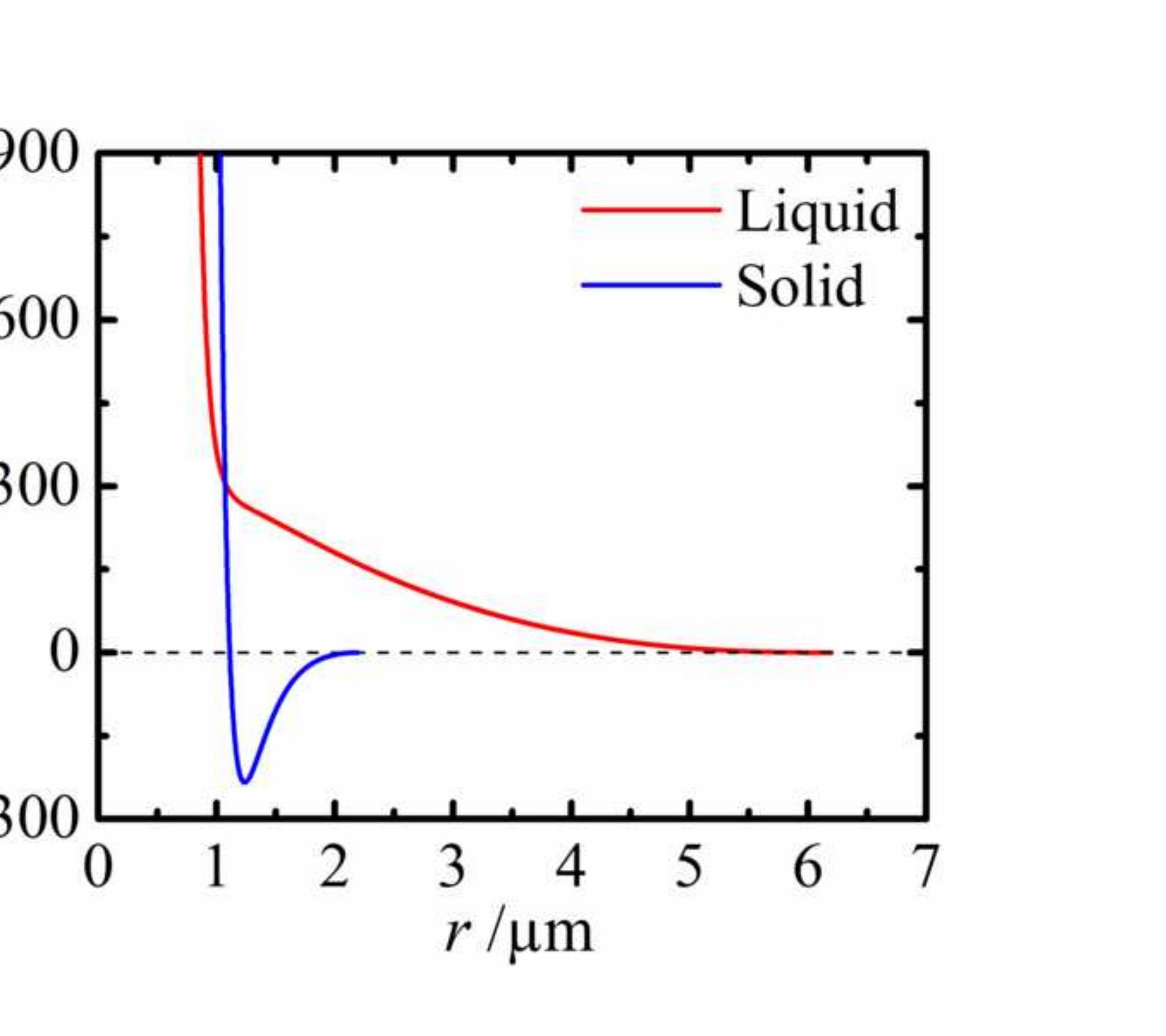


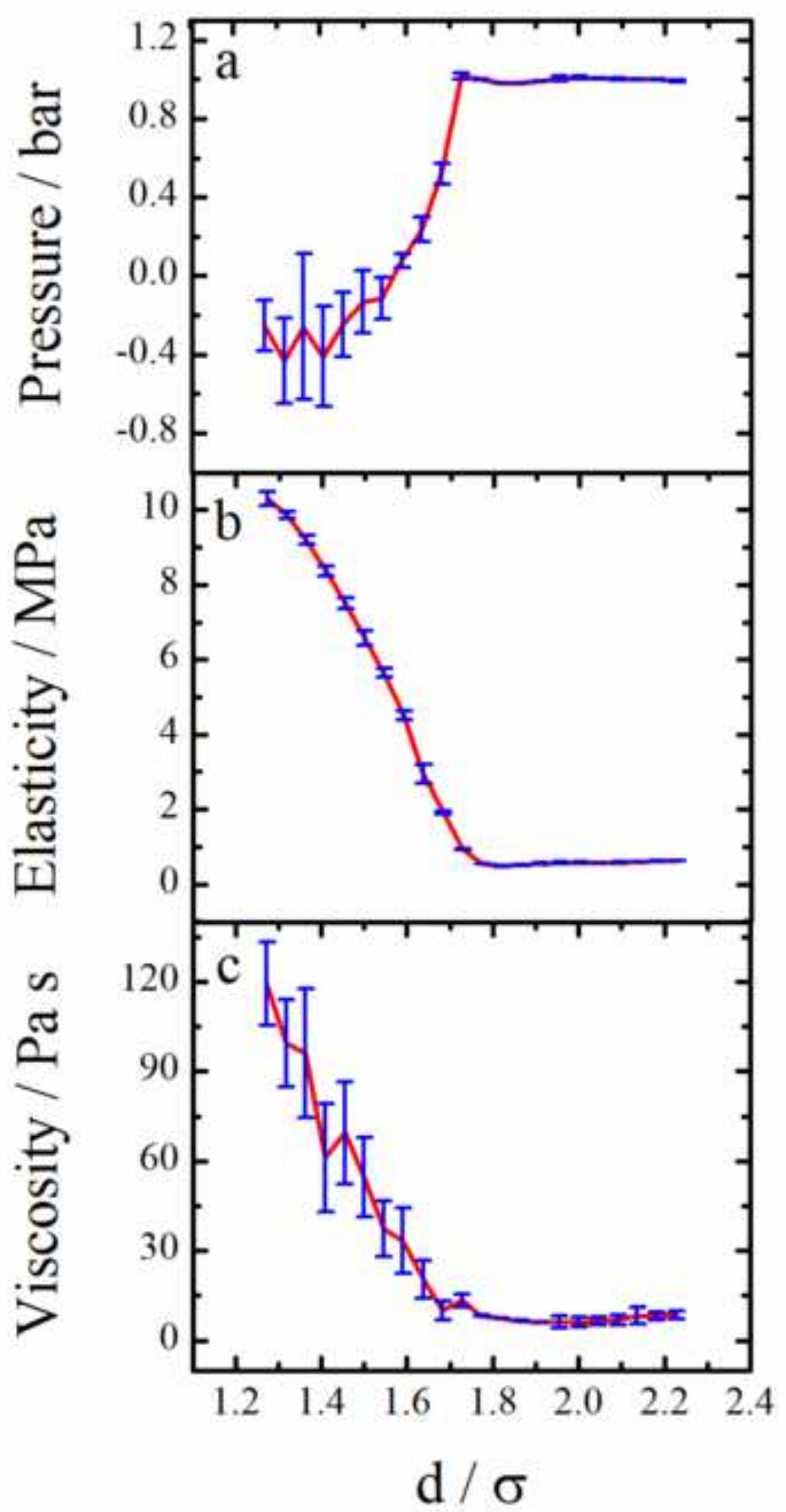

FIG2 

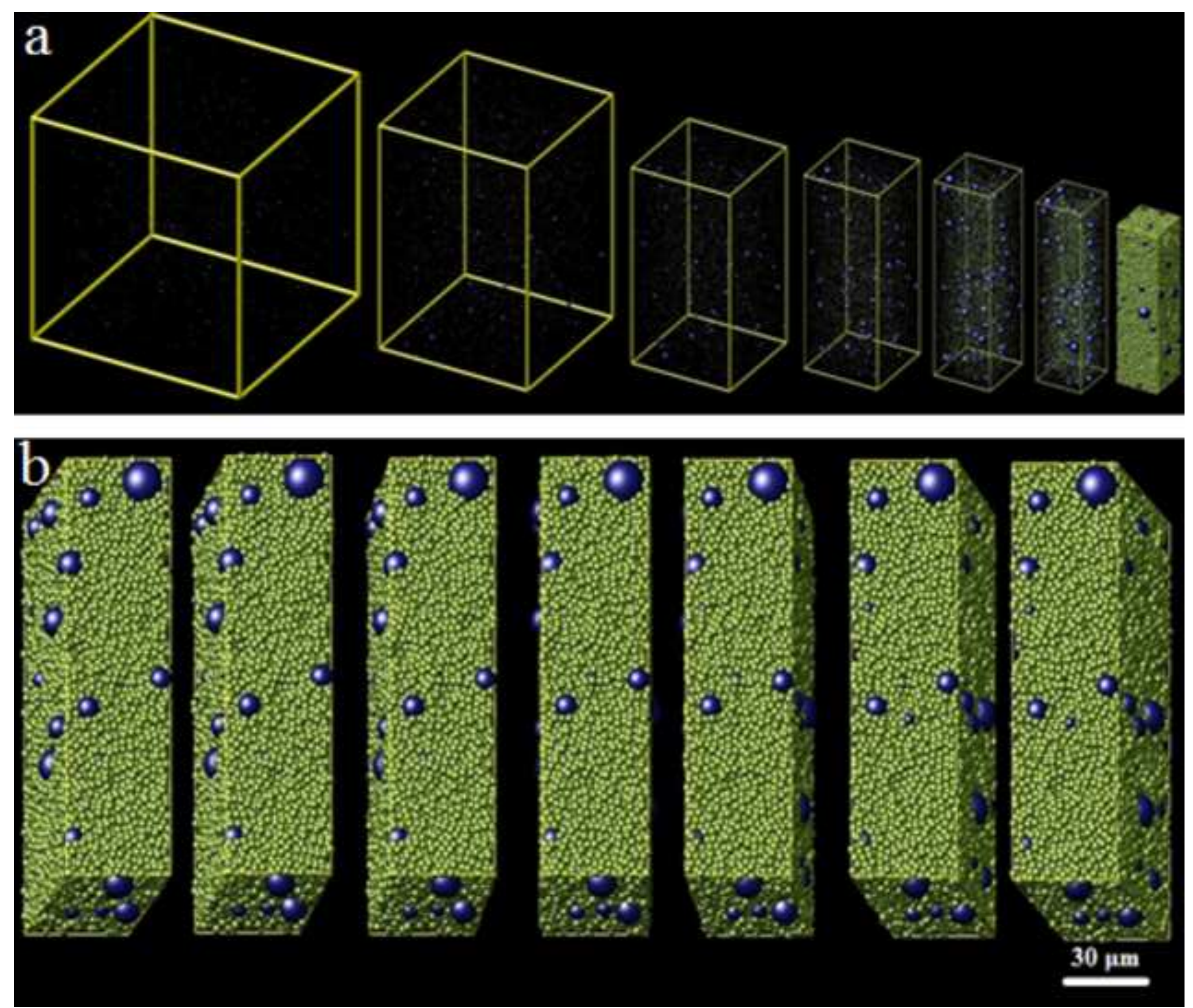

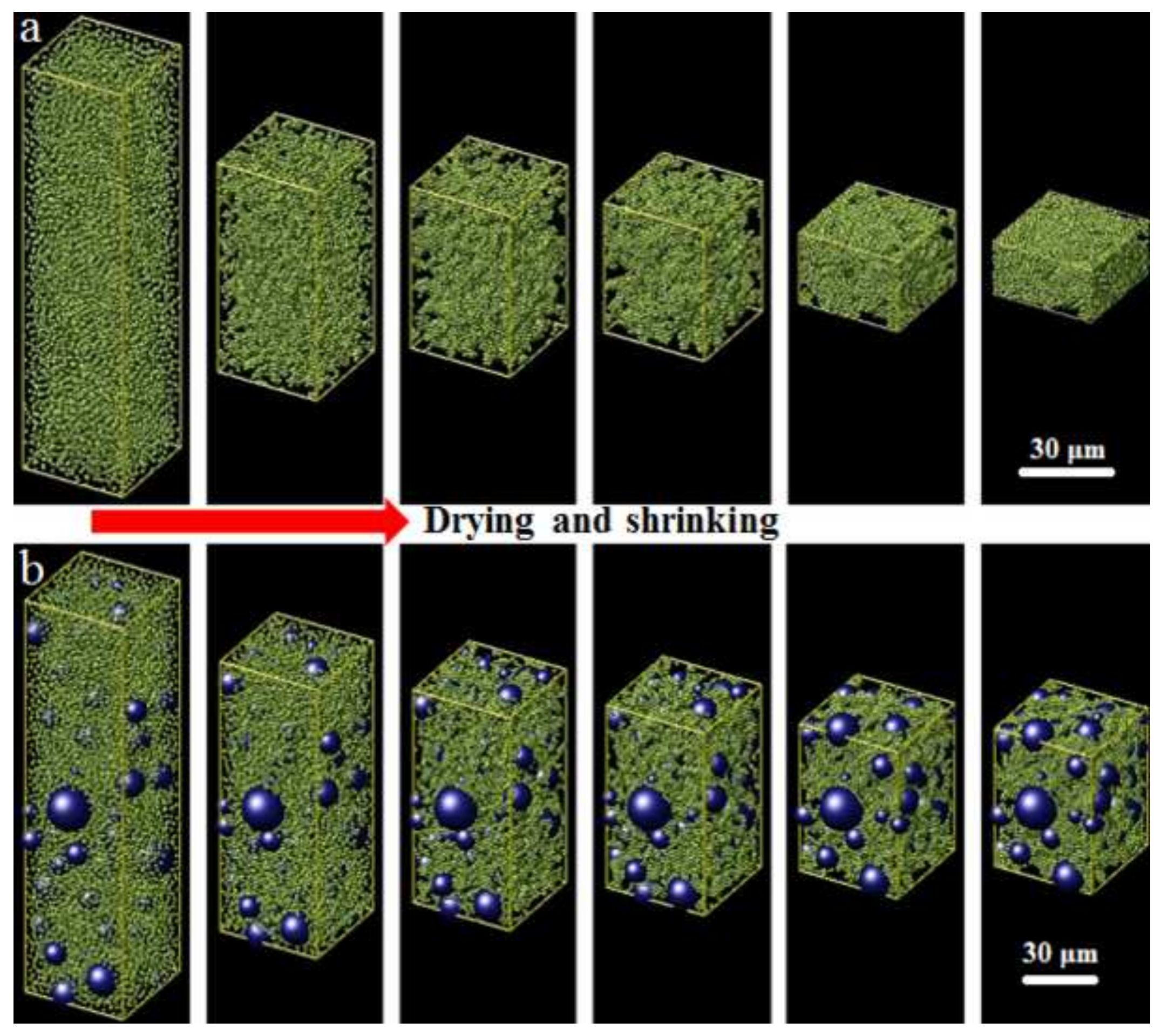

\section{Drying and shrinking}
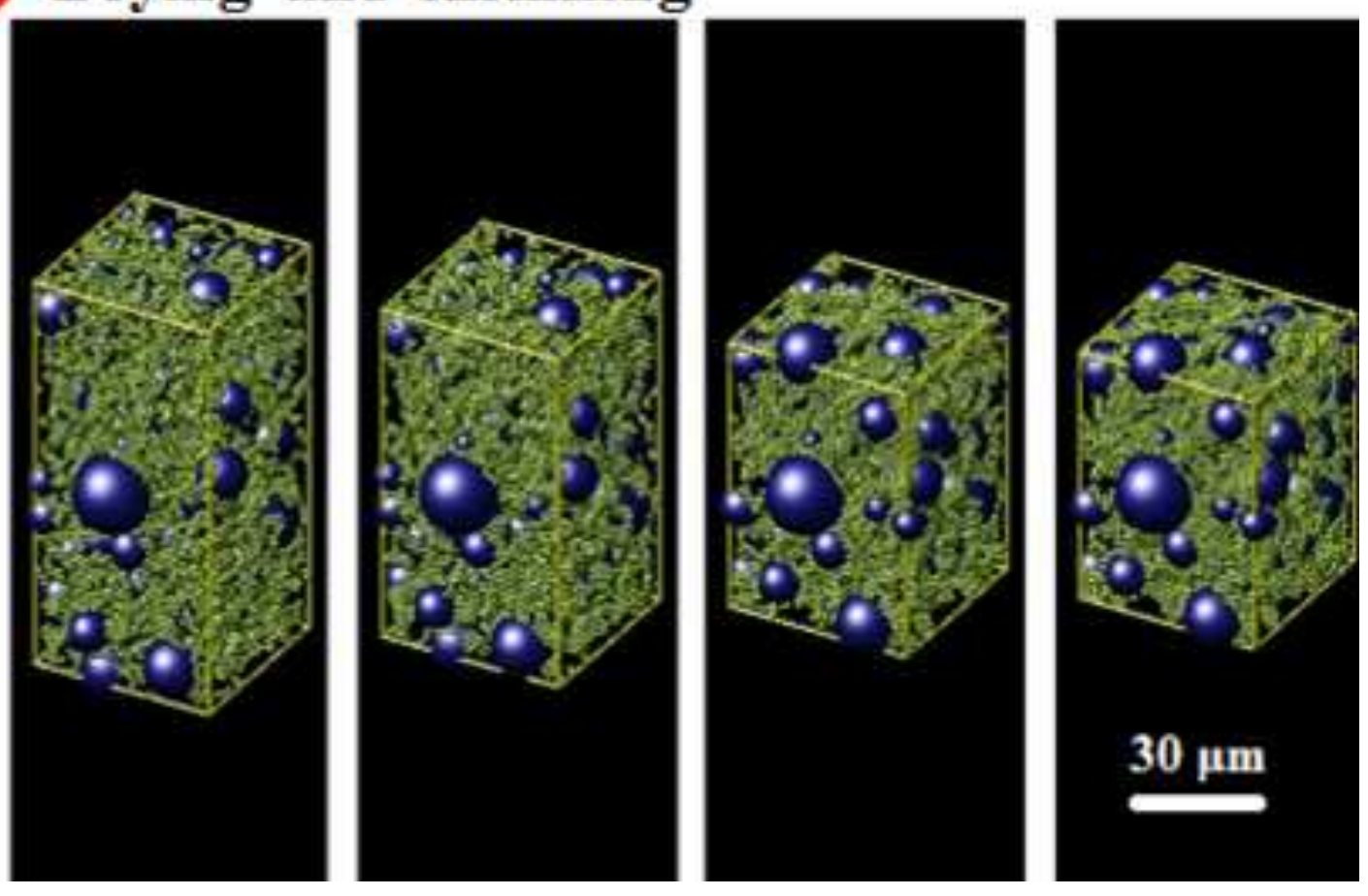


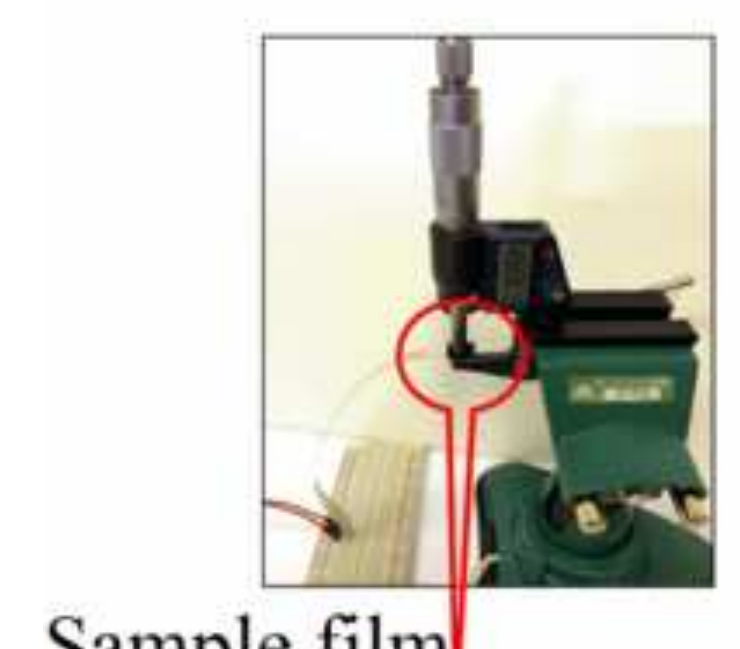

Sample film

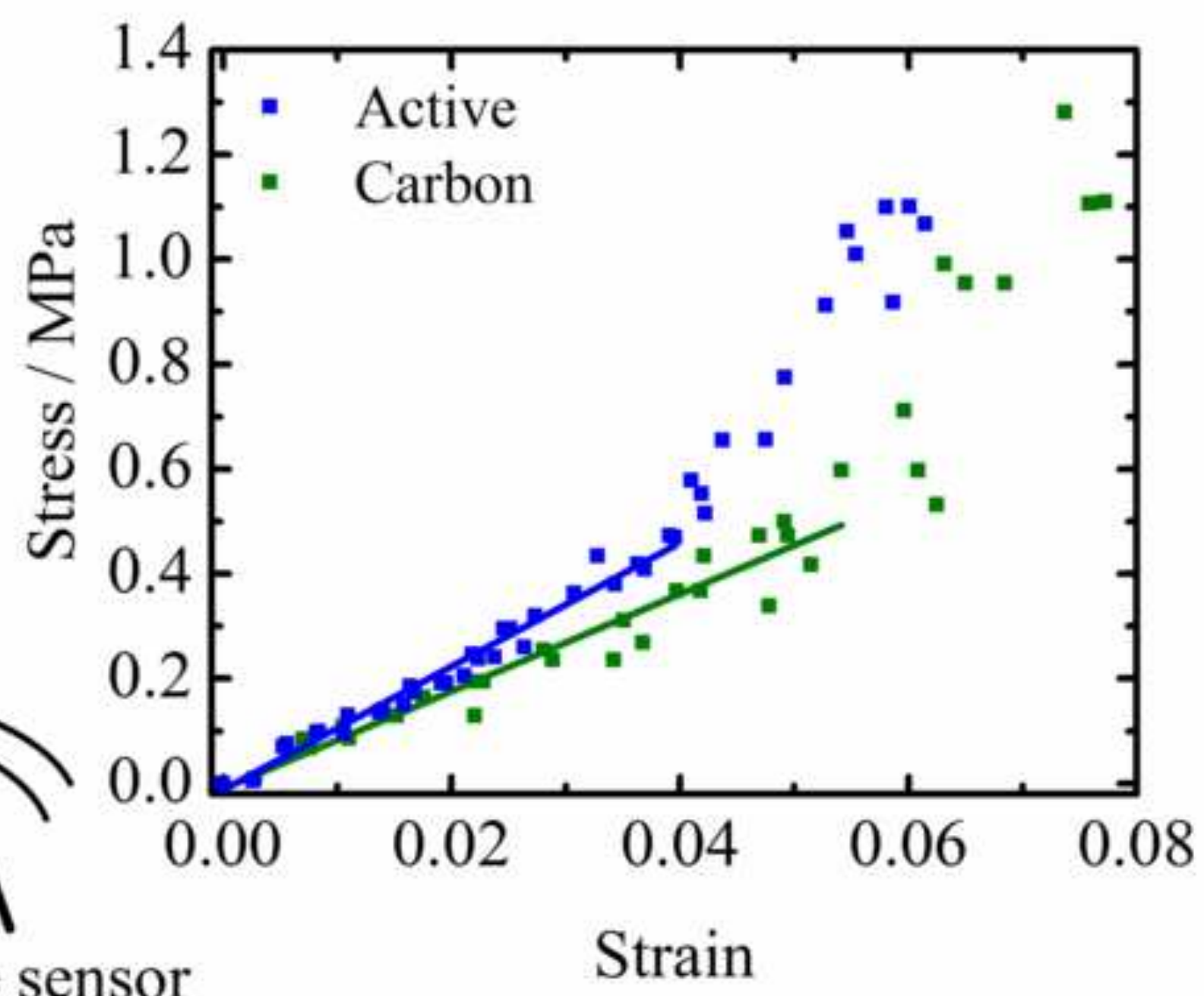

Micrometer

Force sensor

Strain 

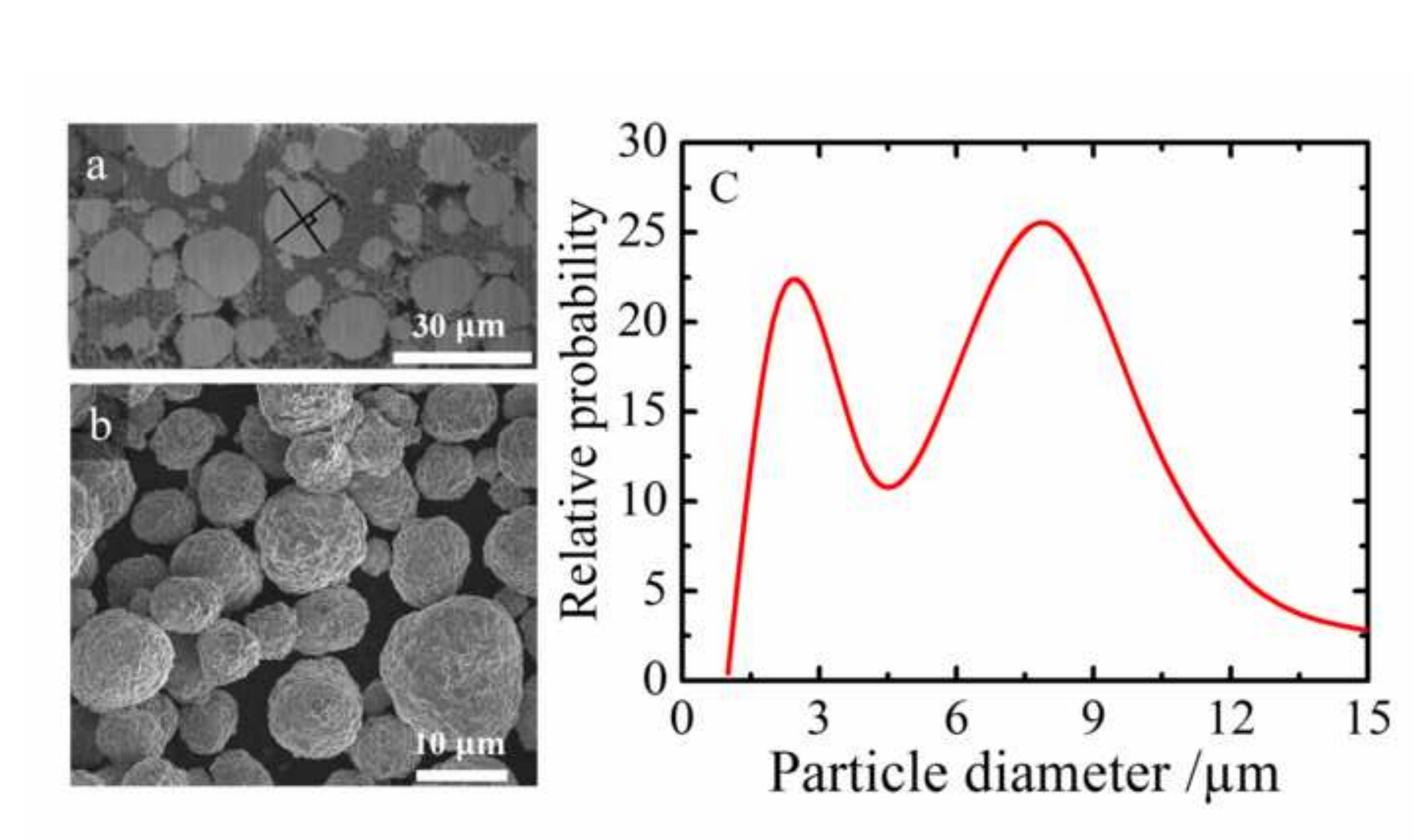

o.jm Particle diameter $/ \mu \mathrm{m}$

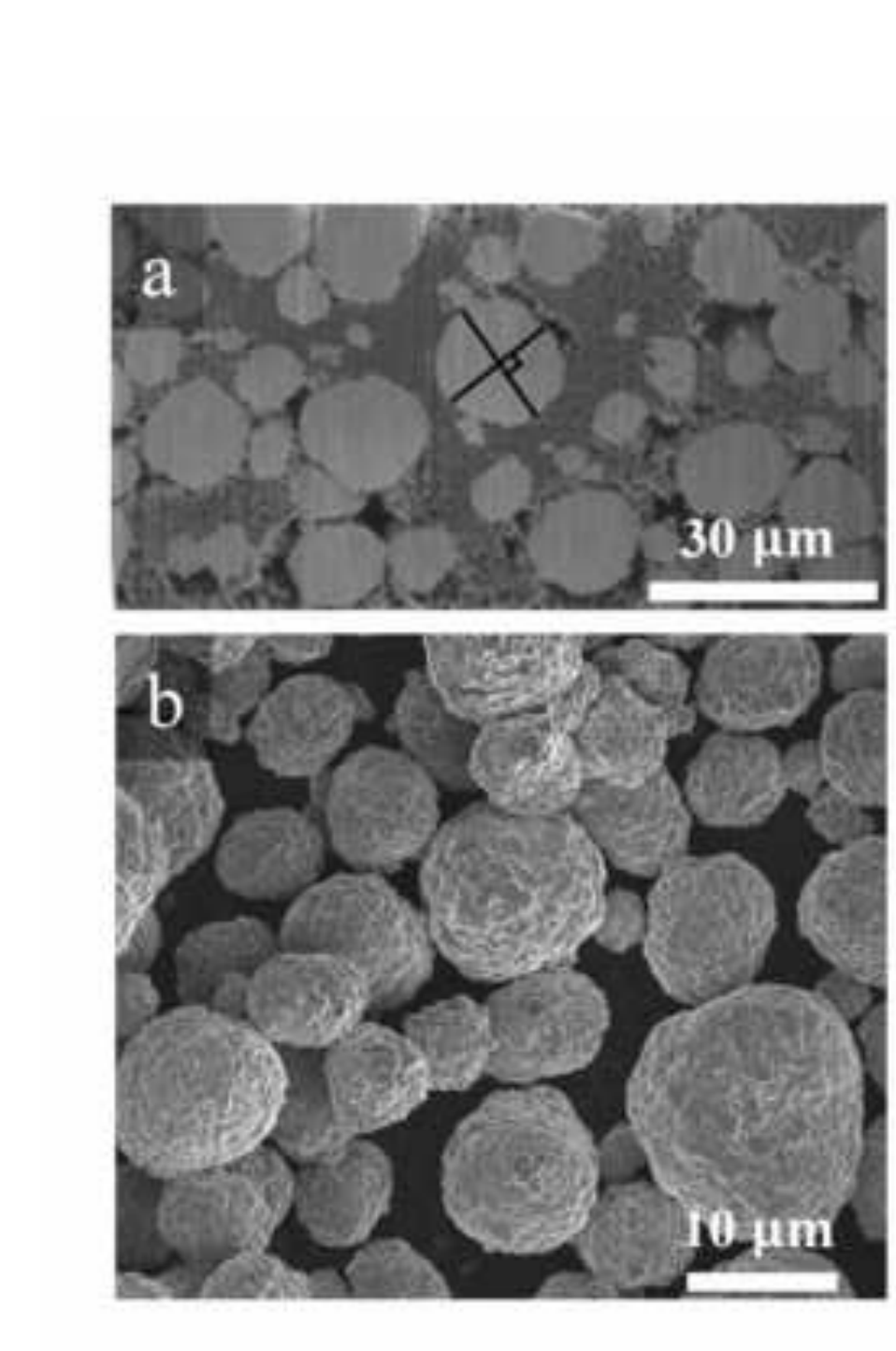

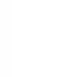

更

更

(1)

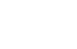




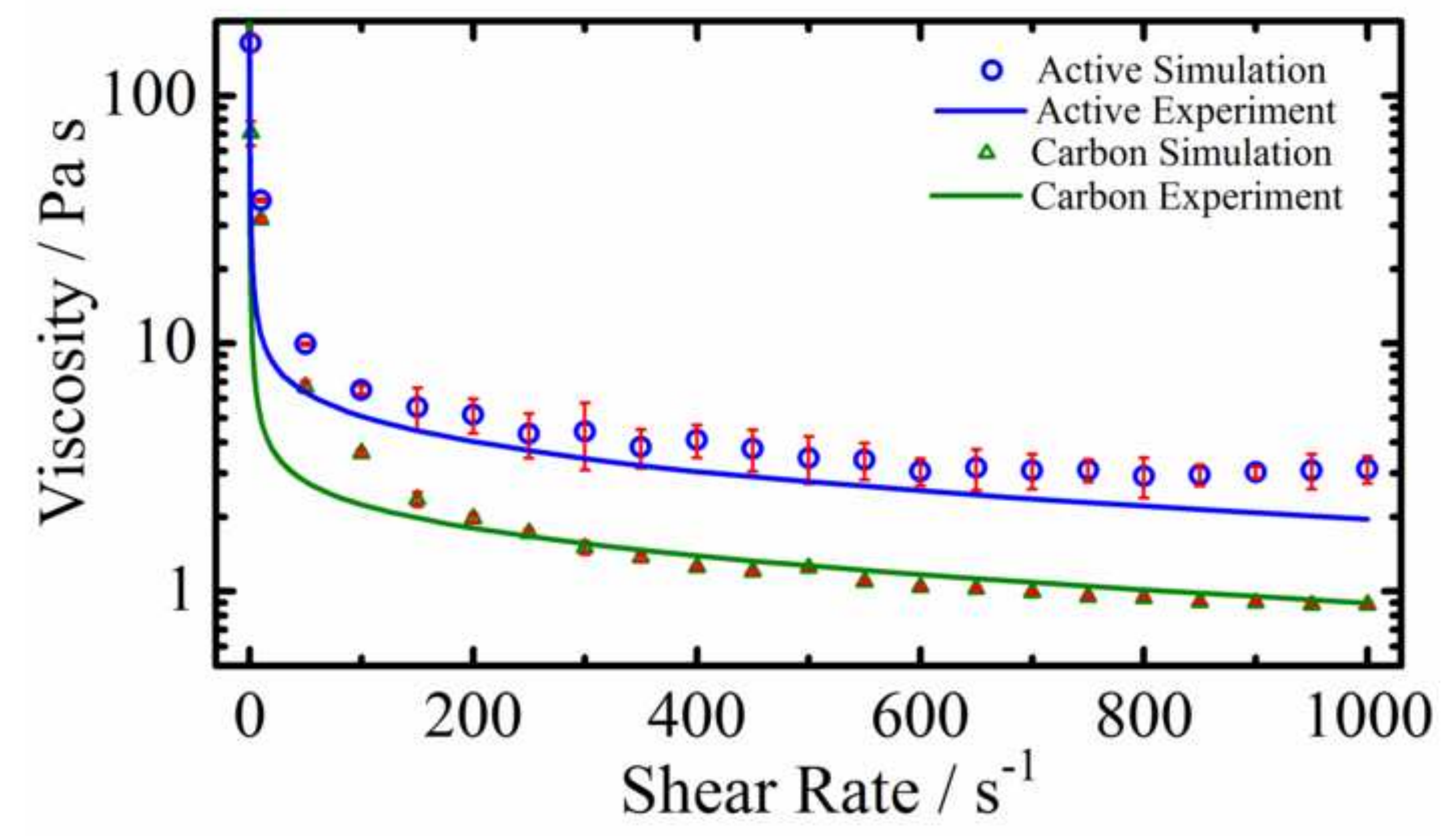



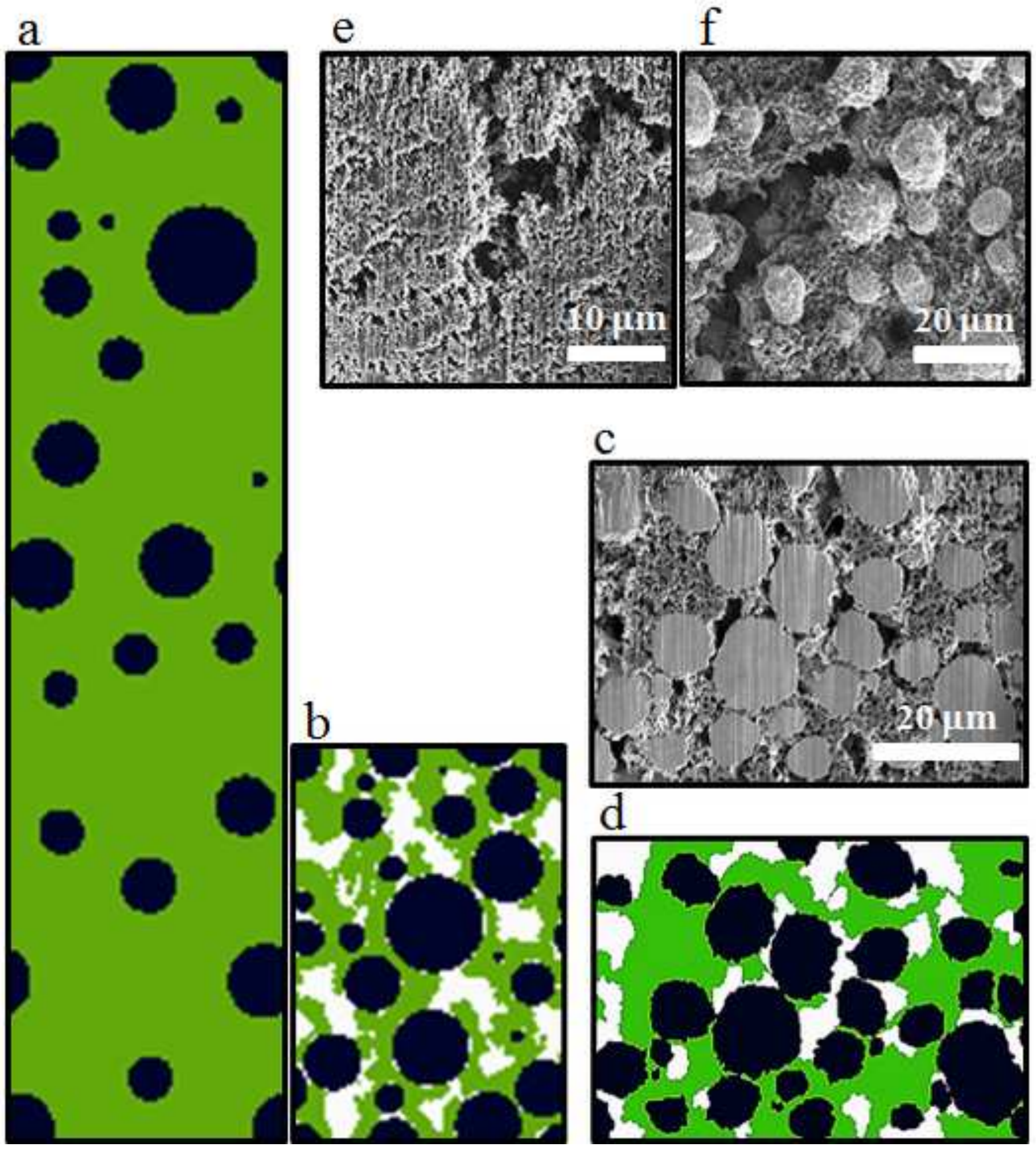
Figure 1: CBD potential as a function of interparticle distance for liquid slurry simulation (red line) and dried solid simulation (blue line). The liquid potential is mostly granular Hertzian in shape and the solid potential is mostly Lennard-Jones. This illustration ignores friction and other dissipative forces.

Figure 2: The effect of changing granular diameter of CBD particles on simulated properties, while holding other parameters and box volume constant: (a) pressure, (b) elasticity (Young's Modulus), and (c) viscosity. Error bars (some smaller than symbol size) show $95 \%$ confidence intervals from multiple simulations. Lines are a guide to the eye.

Figure 3: Rendering of sequential configurations during LAMMPS simulations: (a) equilibration, in which an initially large box shrinks to make a stable slurry; (b) shearing, in which a constantvolume box is deformed to compute the shear stress and hence viscosity. Blue represents active particles and the light green represents CBD particles. Part a is meant to be illustrative and the sequential boxes have a changing size scale, with box sizes shrinking more than is shown upon going from left to right. Shown here are elongated boxes, though most runs used a cubic box.

Figure 4: Drying steps (from stable slurry structure to a stable dried film structure) from LAMMPS simulation: (a) carbon model, (b) active model. Active particles are blue and CBD particles are green.

Figure 5: a) Apparatus used to measure force (stress) and strain of the delaminated electrode film; b) stress-strain graph. Green symbols indicate carbon films and blue symbols indicate active films. Lines indicate the initial slope of stress-strain graph (Young's modulus).

Figure 6: (a) A Typical cross-sectional FIB/SEM image of uncalendered cathode containing spherical Toda 523 particles for particle size distribution purposes; (b) an SEM image of active particle powder for active particle size distribution validation purposes; (c) Experimental size distribution of Toda NCM-523 active material, showing particle number probability vs. effective diameter.

Figure 7: Viscosity of slurry at different shear rates for pure carbon (green), and active sample (blue) from experiment (line), and simulation (symbols). In some cases error bars are smaller than symbol sizes. Lines are used for experiments due to the close spacing of the data.

Figure 8: Microstructure comparison by randomly picked 2D cross-sections: (a) simulated slurry, (b) simulated dried film, (c) SEM/FIB of dried film, and (d) segmentation of SEM/FIB. In the segmented images active particles are blue, CBD is green, and macropores are white. All images have the same scale as given in (c). (e) SEM images of dried but uncalendered carbon sample and (f) active sample, showing mesoscale pores and aggregates of CBD. FIB planarization was used on (e), but not (f). 


\section{Microstructure generated by the particle-based simulation}

Carbon, binder, nanopores(CBD)

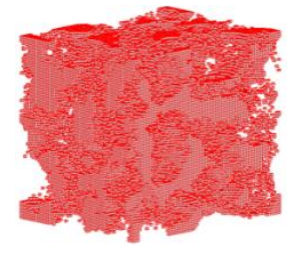

Macropores

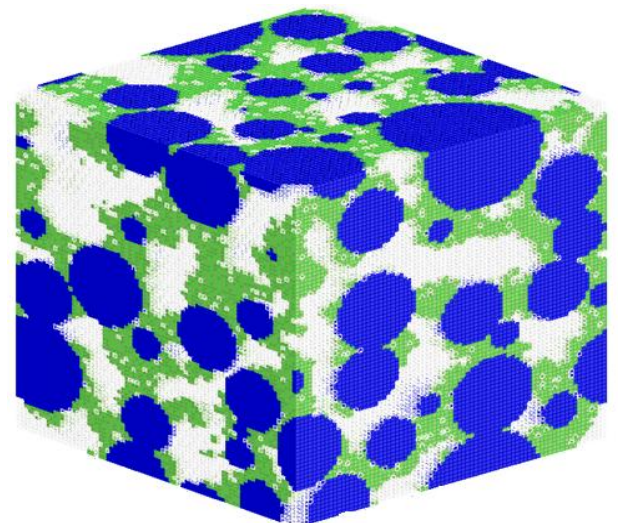

Carbon domain(green) Active particle(blue) Macropores (white) 